\title{
Silver-catalyzed synthesis of diaryl selenides by reaction of diaryl diselenides with aryl boronic acids
}

Bruna Goldani, Vanessa G. Ricordi, Natália Seus, Eder J. Lenardão, Ricardo F. Schumacher, Diego Alves*

LASOL - CCQFA - Universidade Federal de Pelotas - UFPel - P.O. Box 354 - 96010900, Pelotas, RS, Brazil.

diego.alves@ufpel.edu.br 


\section{SELECTED SPECTRA}
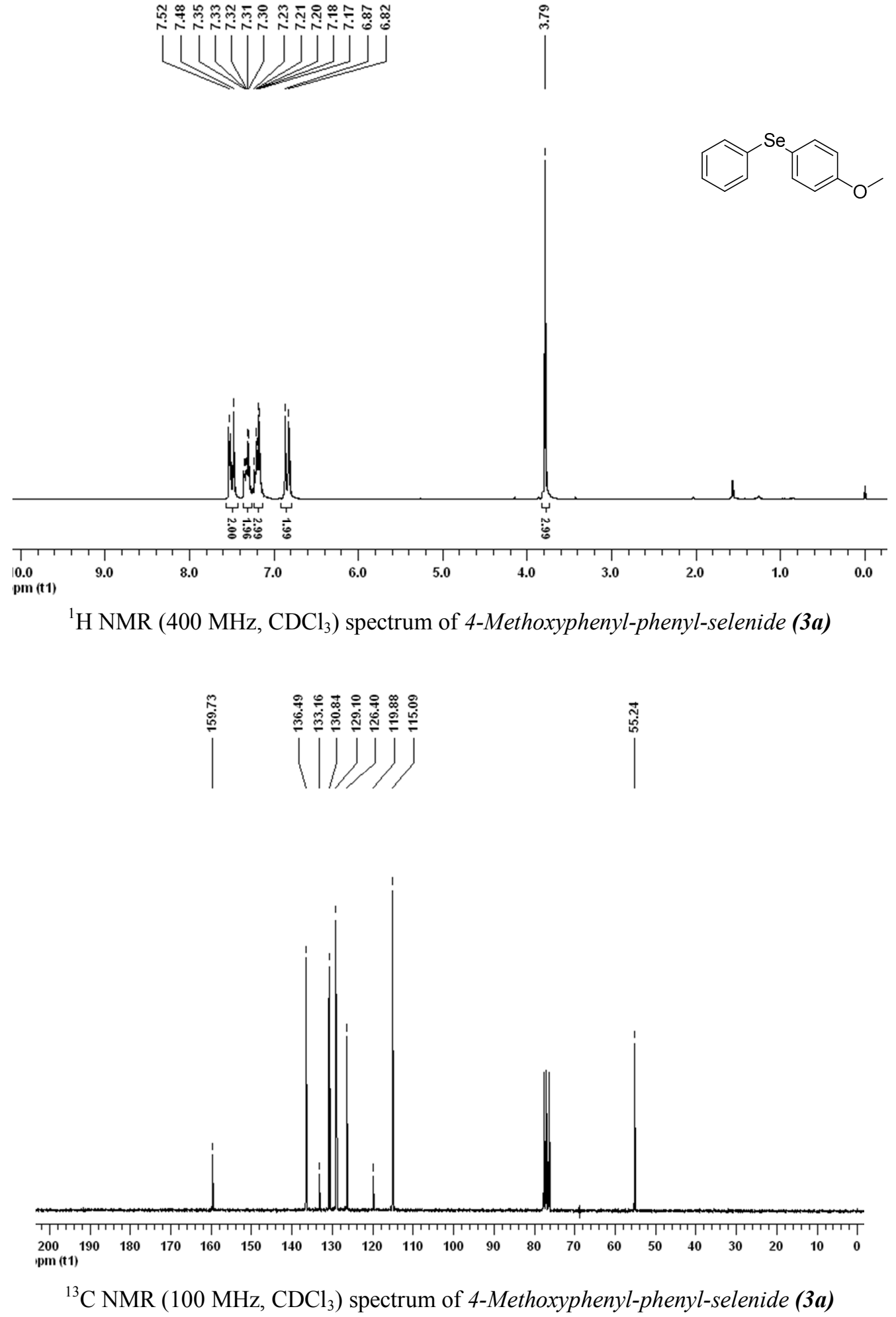


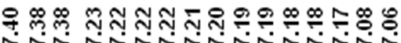
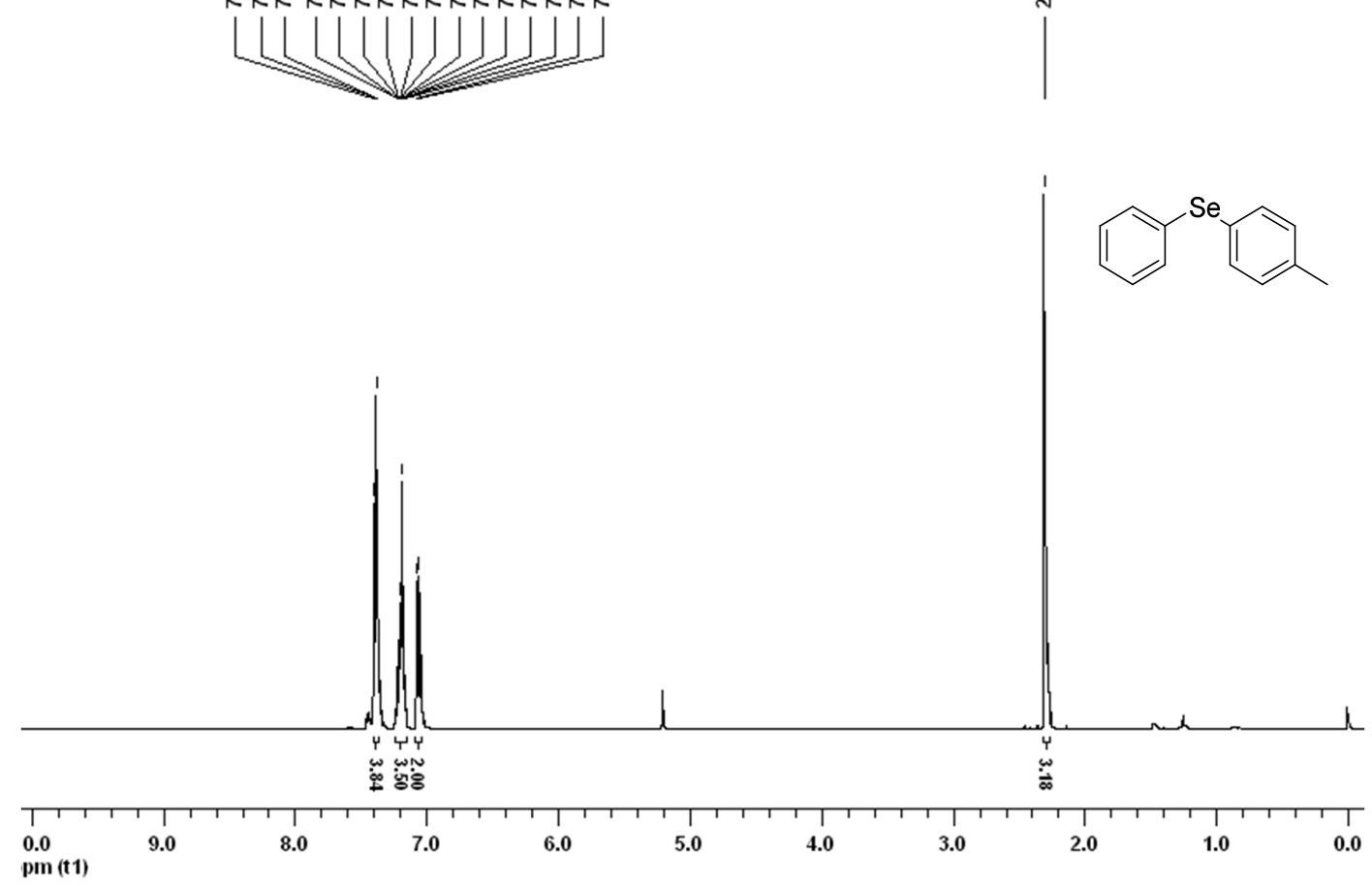

${ }^{1} \mathrm{H}$ NMR (400 MHz, $\mathrm{CDCl}_{3}$ ) spectrum of 4-Tolyl-phenyl-selenide (3b)

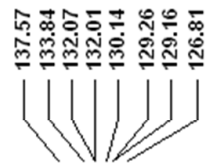

$\stackrel{8}{\stackrel{2}{2}}$

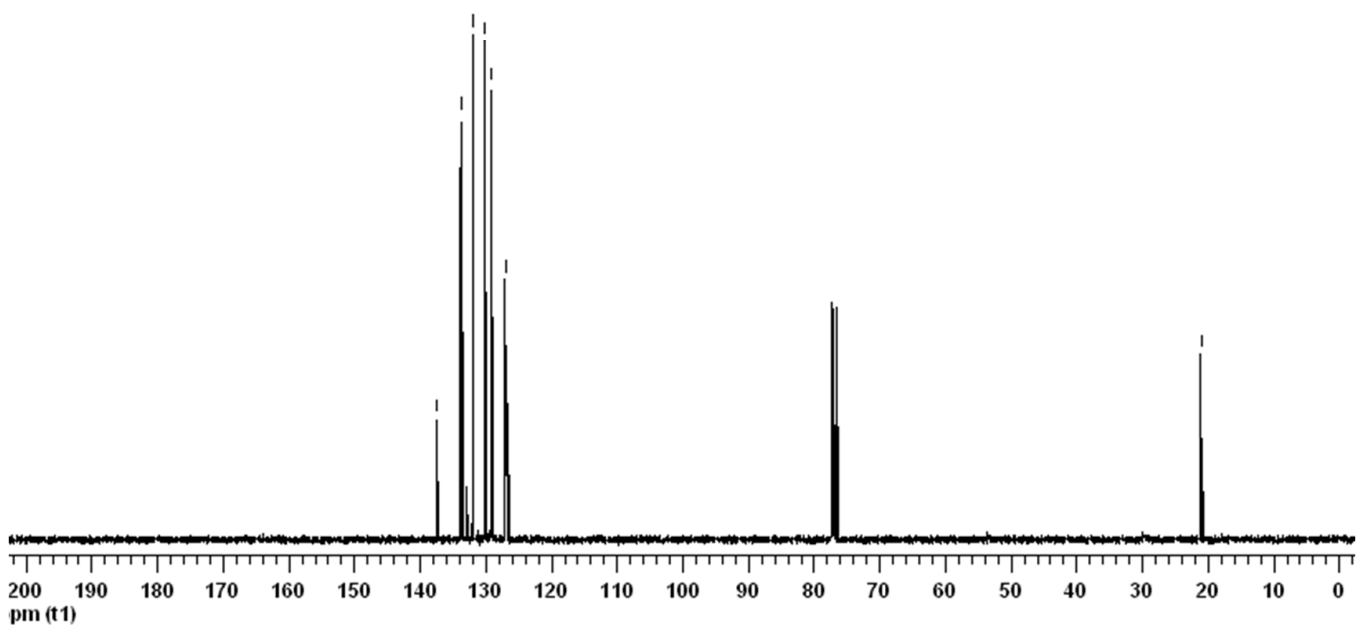

${ }^{13} \mathrm{C}$ NMR (100 MHz, $\mathrm{CDCl}_{3}$ ) spectrum of 4-Tolyl-phenyl-selenide (3b) 


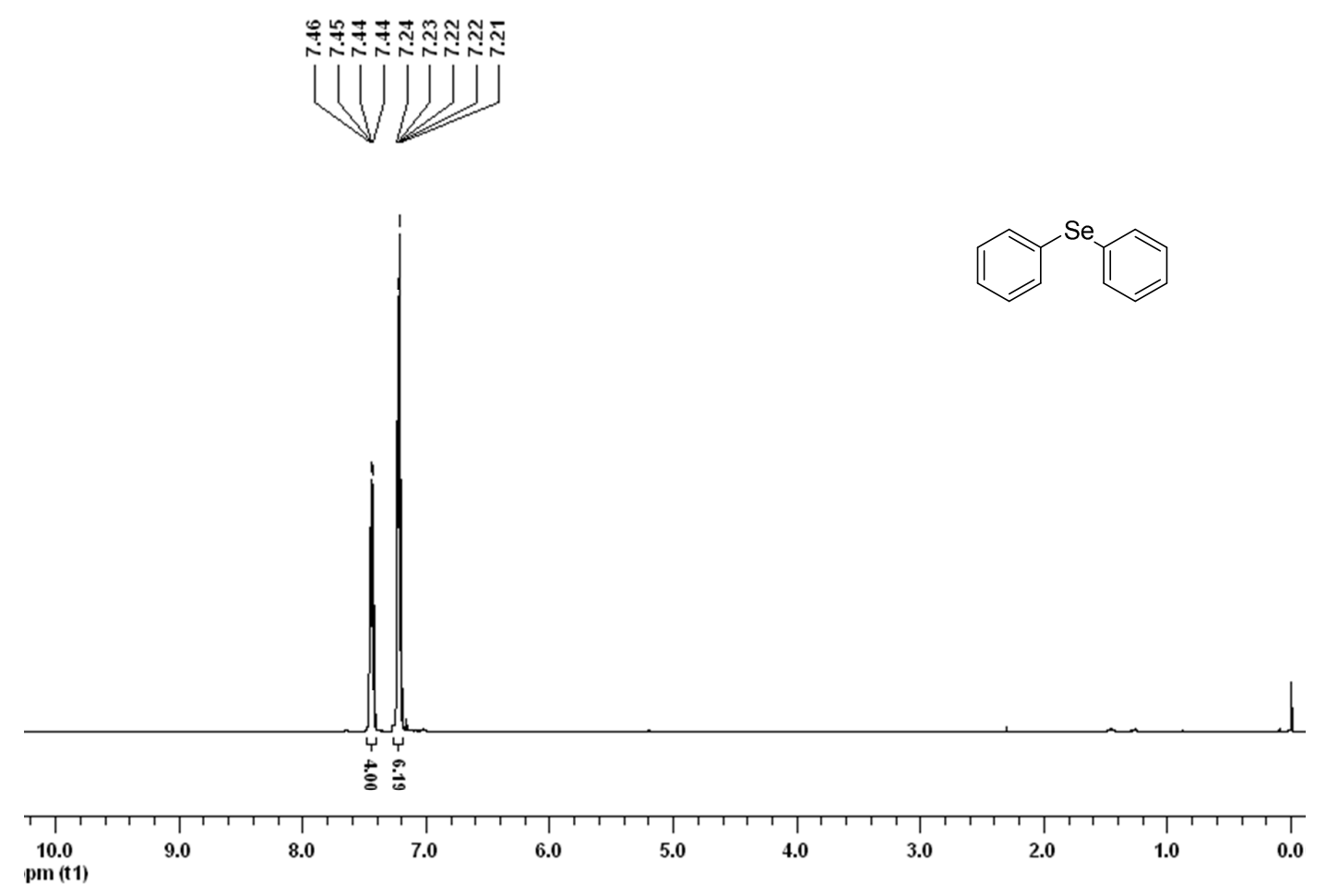

${ }^{1} \mathrm{H}$ NMR (400 MHz, $\mathrm{CDCl}_{3}$ ) spectrum of diphenyl-selenide (3c)

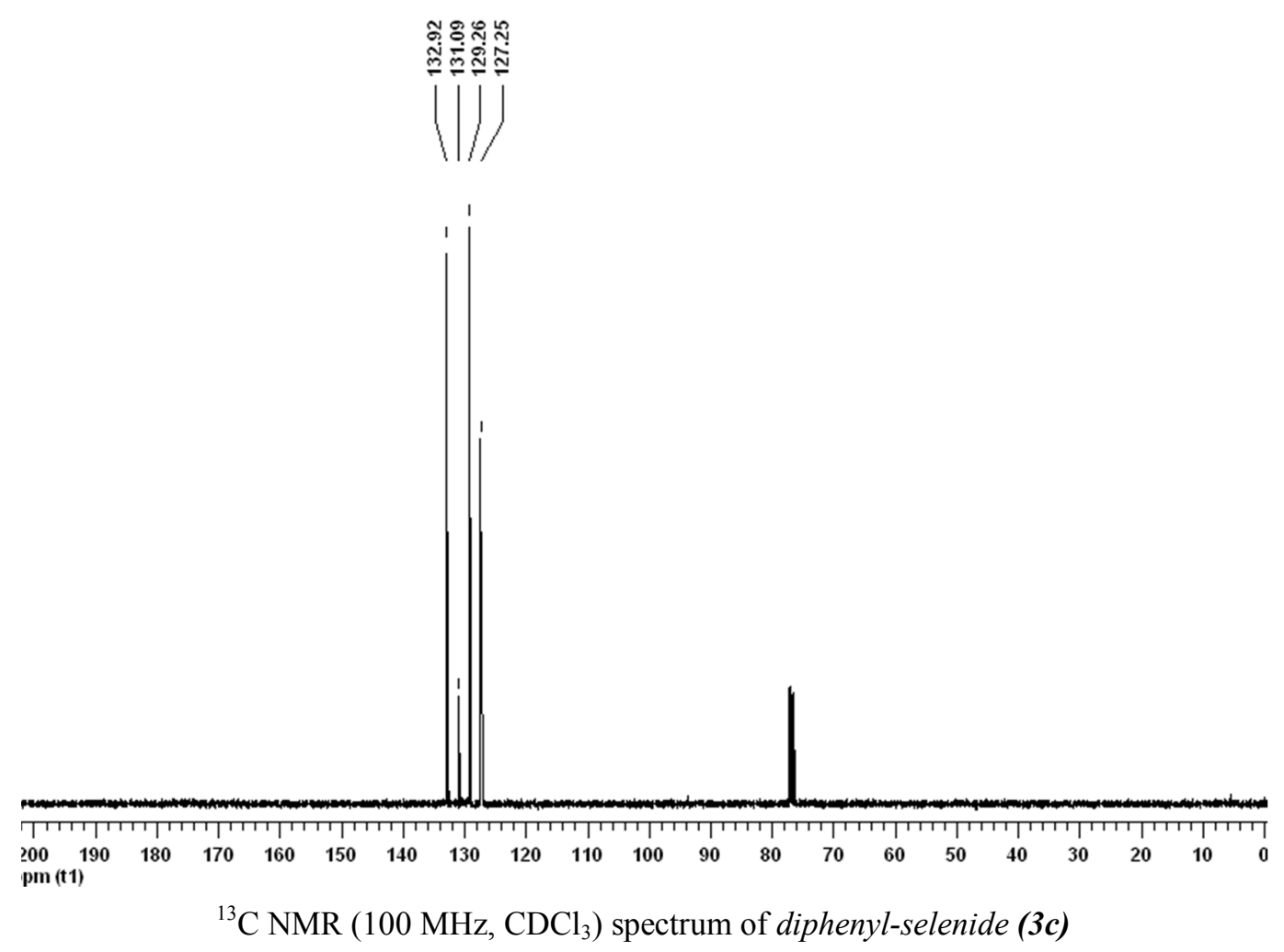




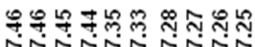
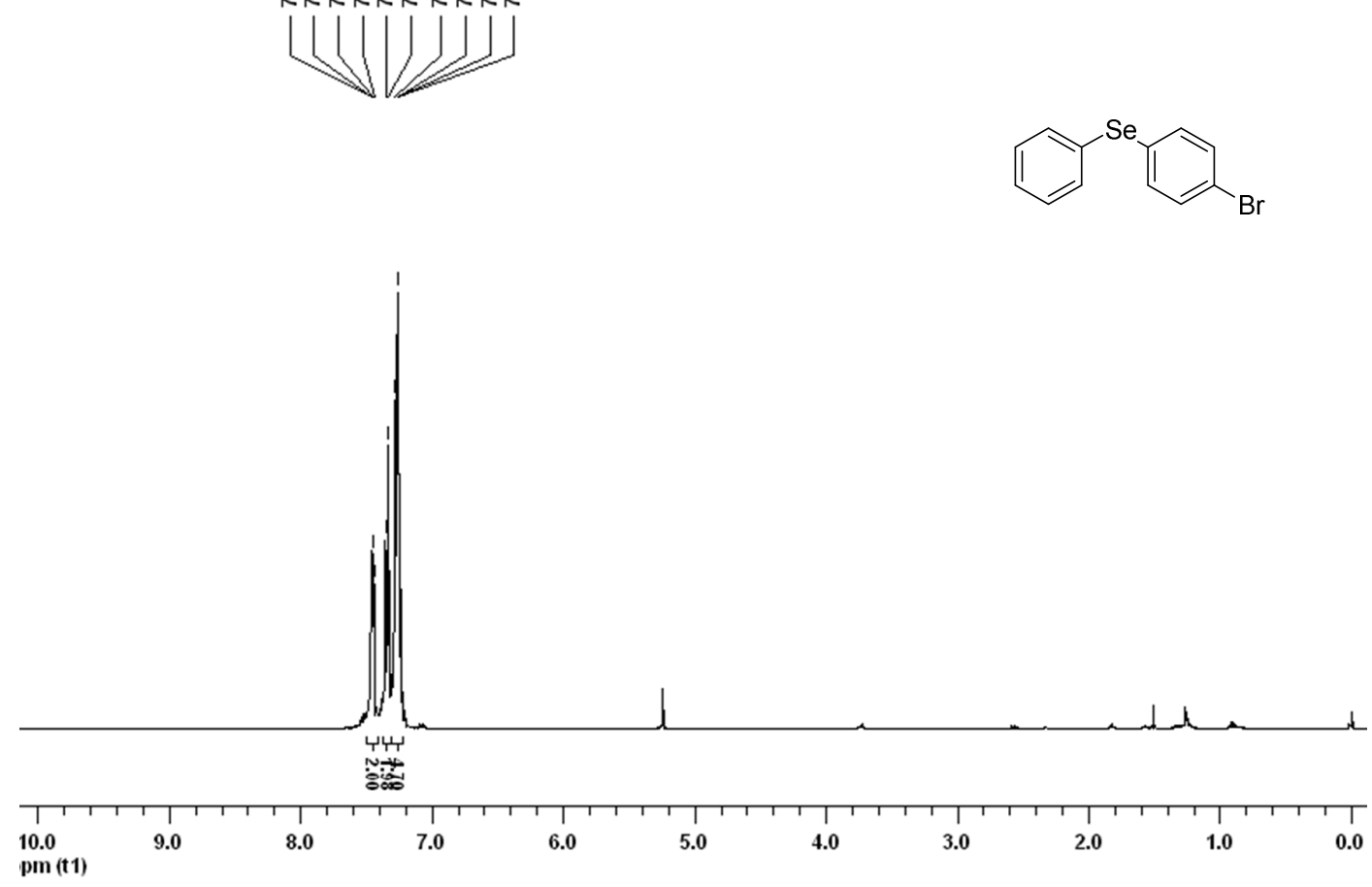

${ }^{1} \mathrm{H}$ NMR (400 MHz, $\mathrm{CDCl}_{3}$ ) spectrum of 4-Bromophenyl-phenyl-selenide (3d)

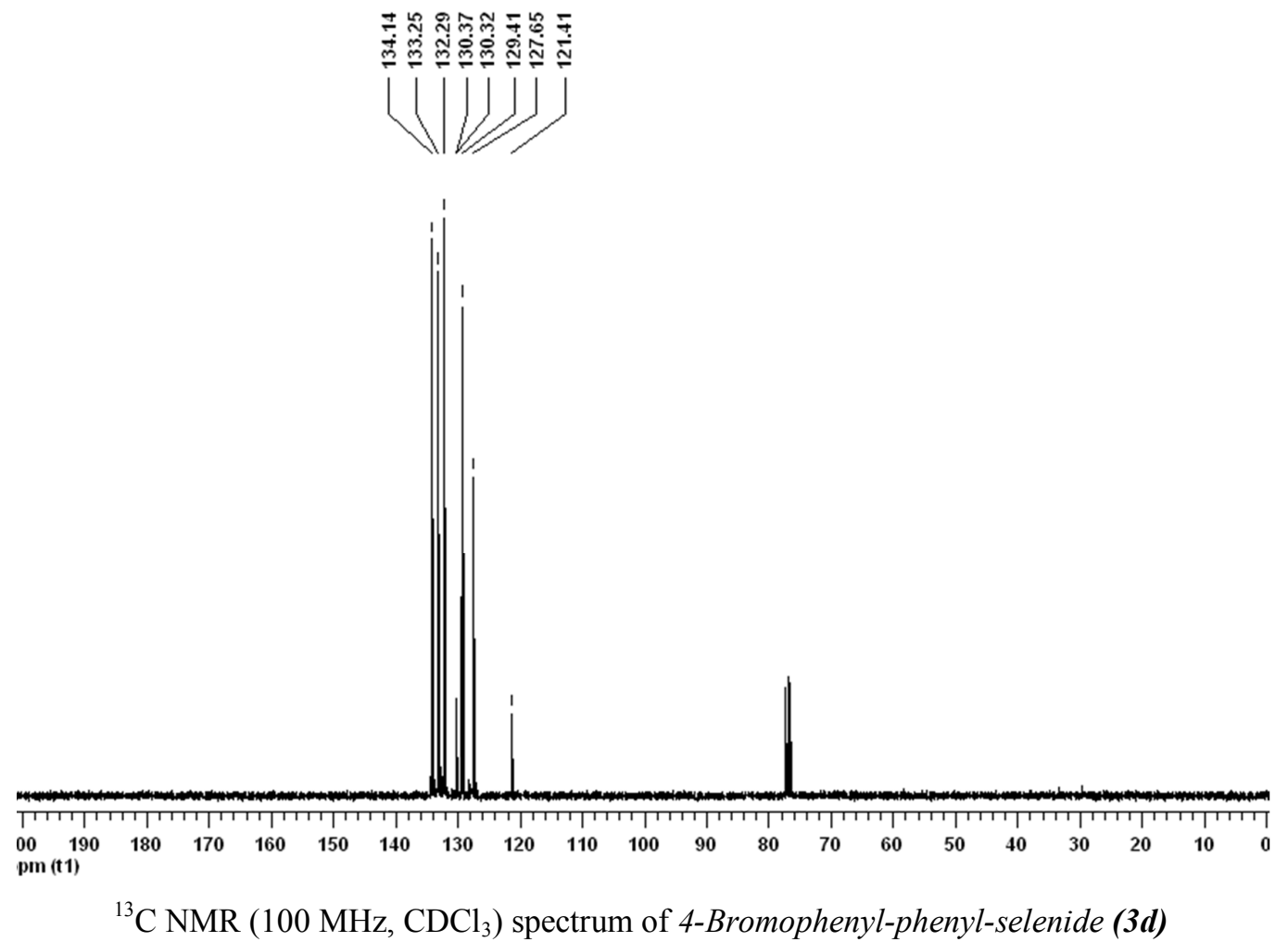




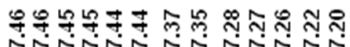
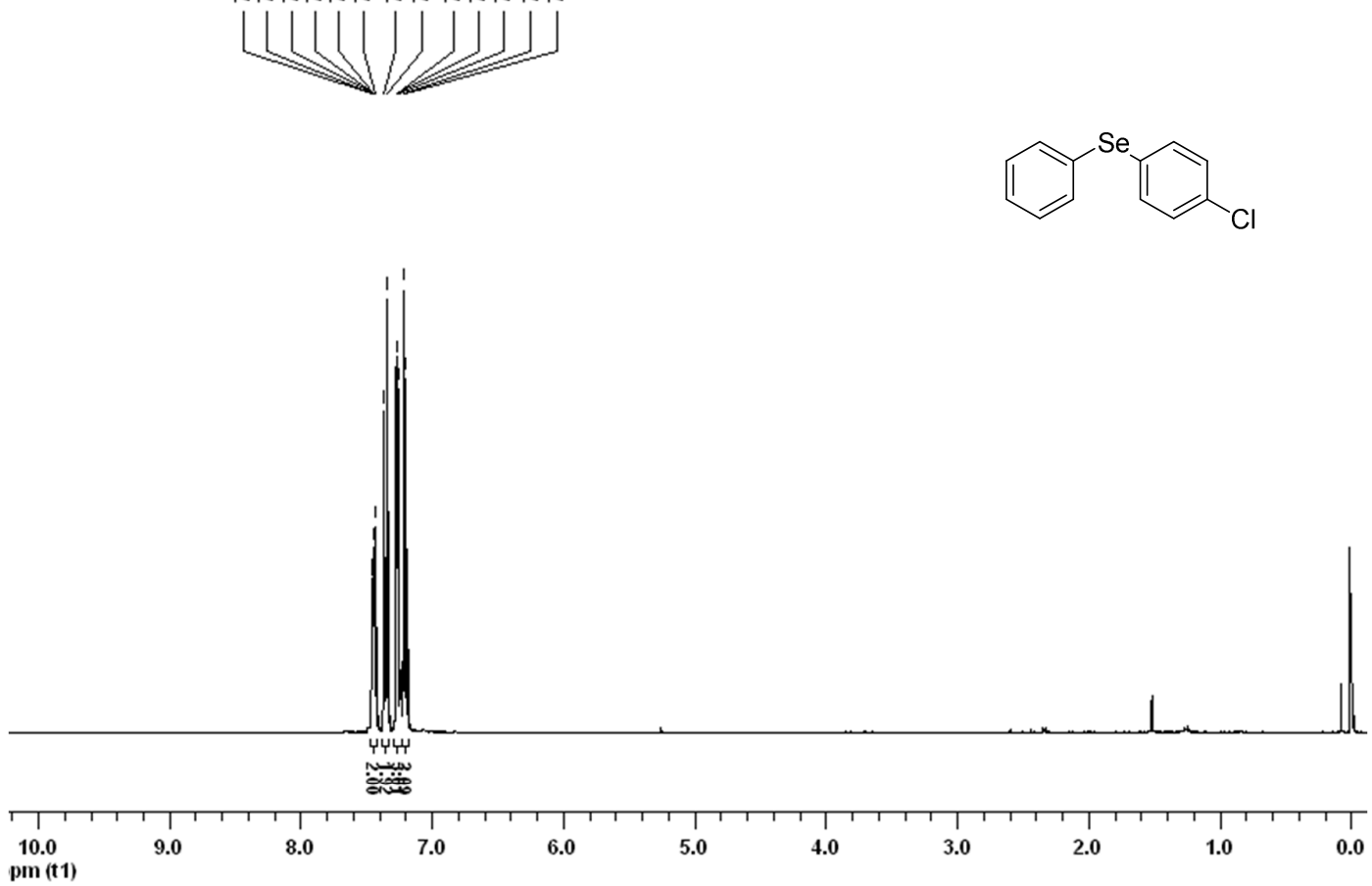

${ }^{1} \mathrm{H} \mathrm{NMR}\left(400 \mathrm{MHz}, \mathrm{CDCl}_{3}\right.$ ) spectrum of 4-Chlorophenyl-phenyl-selenide (3e)

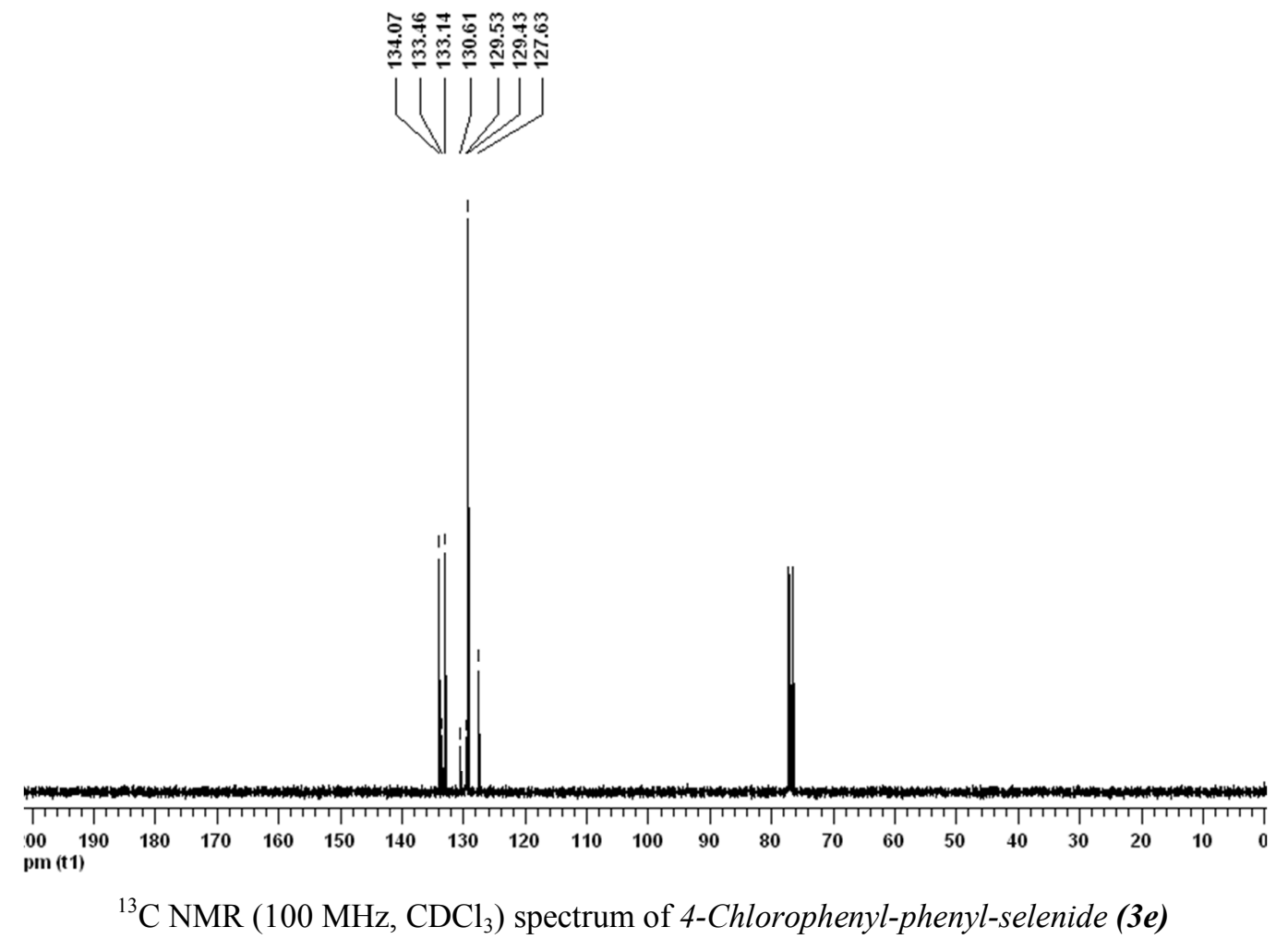




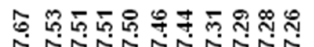
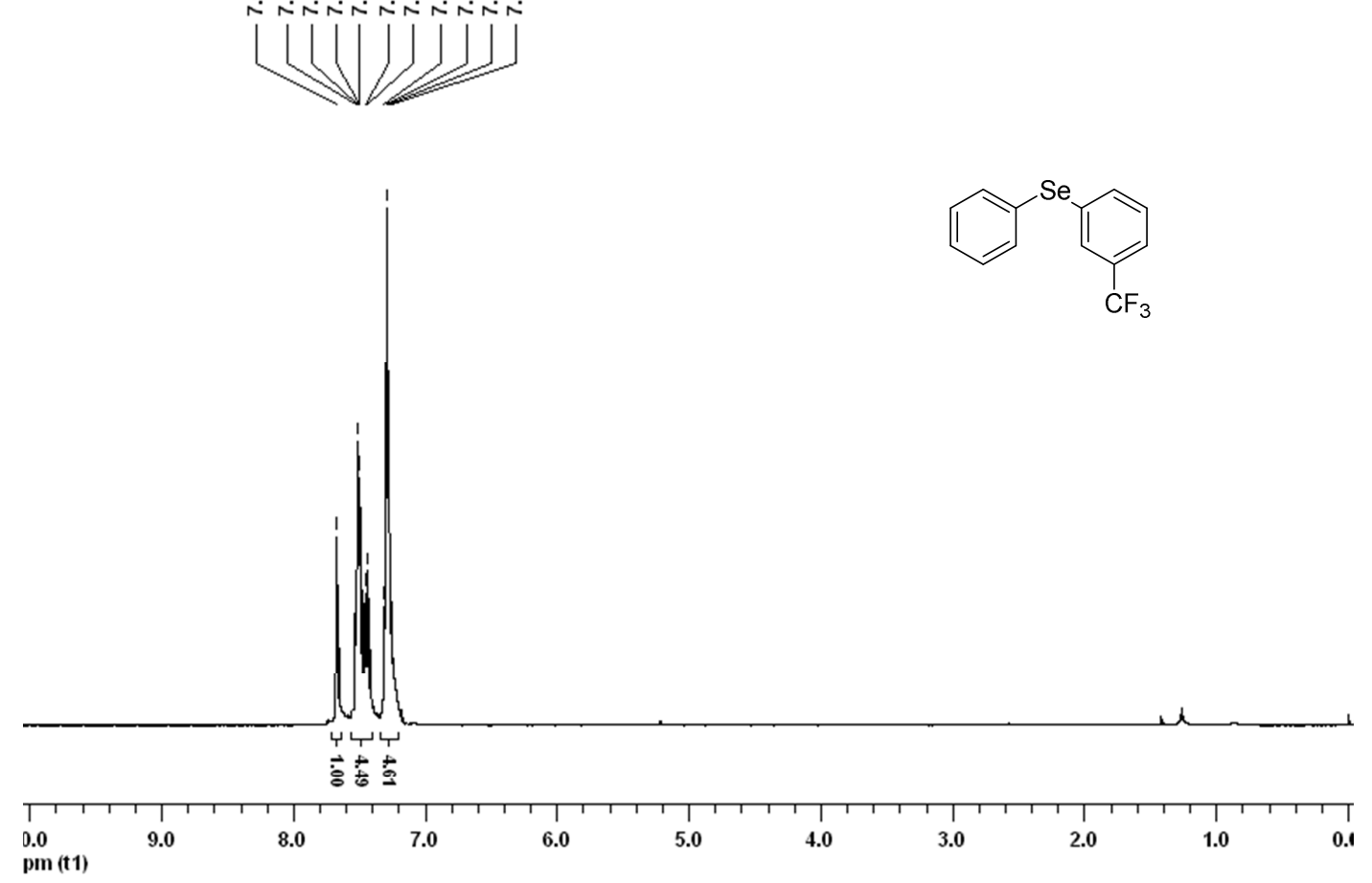

${ }^{1} \mathrm{H}$ NMR (400 MHz, $\mathrm{CDCl}_{3}$ ) spectrum of 3-(trifluoromethyl)phenyl-phenyl-selenide (3f)
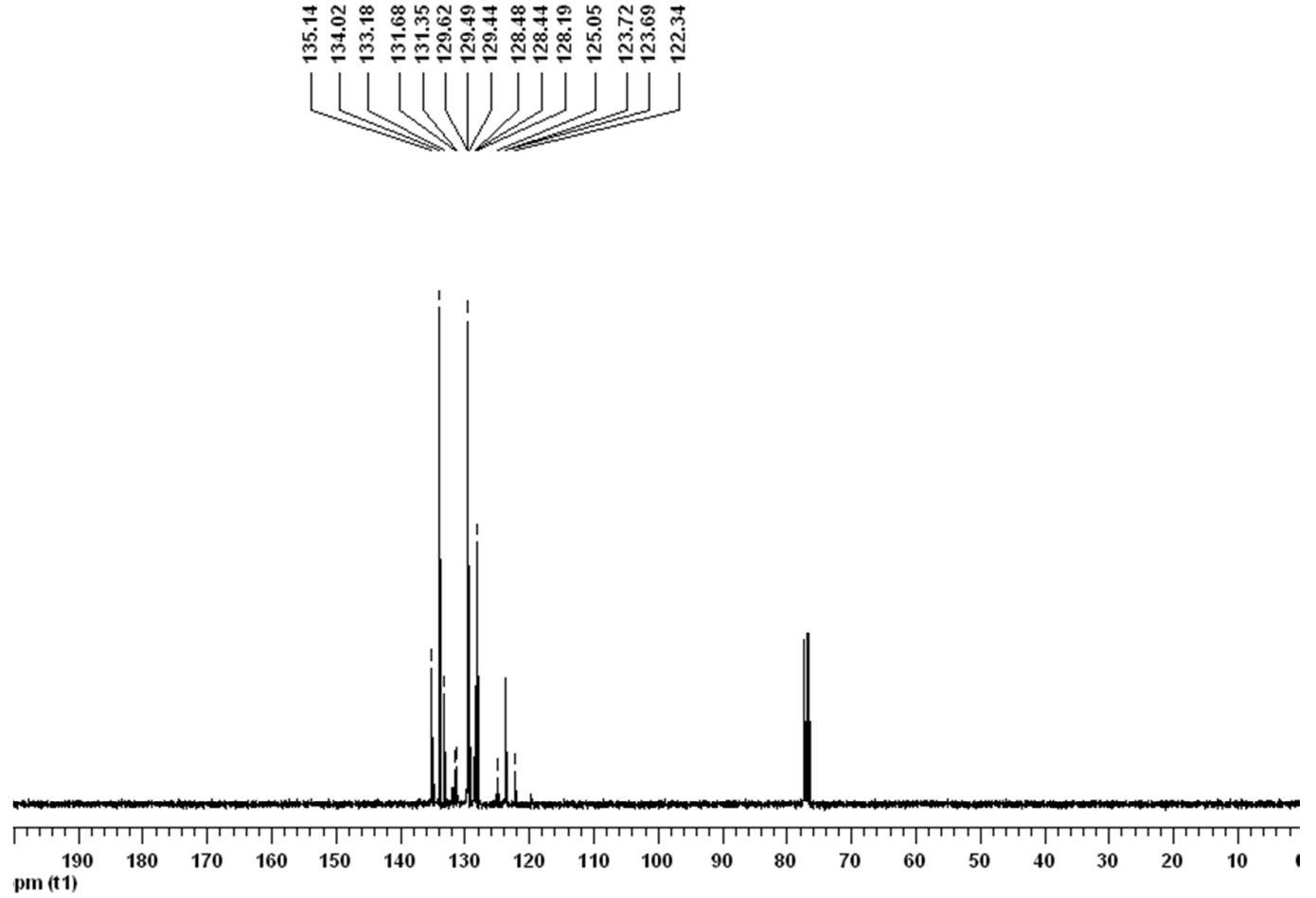

${ }^{13} \mathrm{C} \mathrm{NMR}\left(100 \mathrm{MHz}, \mathrm{CDCl}_{3}\right)$ spectrum of 3-(trifluoromethyl)phenyl-phenyl-selenide (3f) 

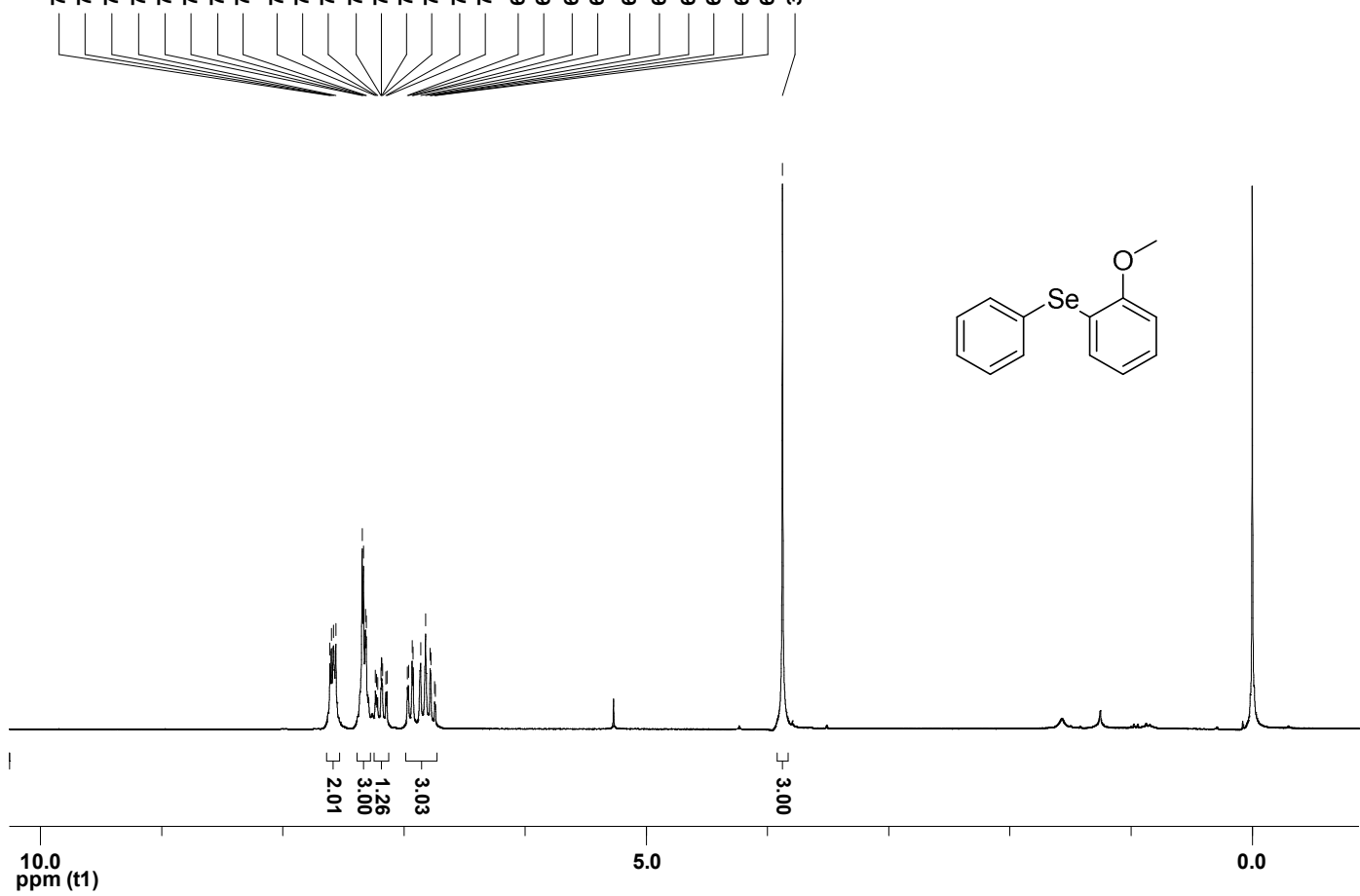

${ }^{1} \mathrm{H} \mathrm{NMR}\left(400 \mathrm{MHz}, \mathrm{CDCl}_{3}\right.$ ) spectrum of 2-Methoxyphenyl-phenyl-selenide (3g)
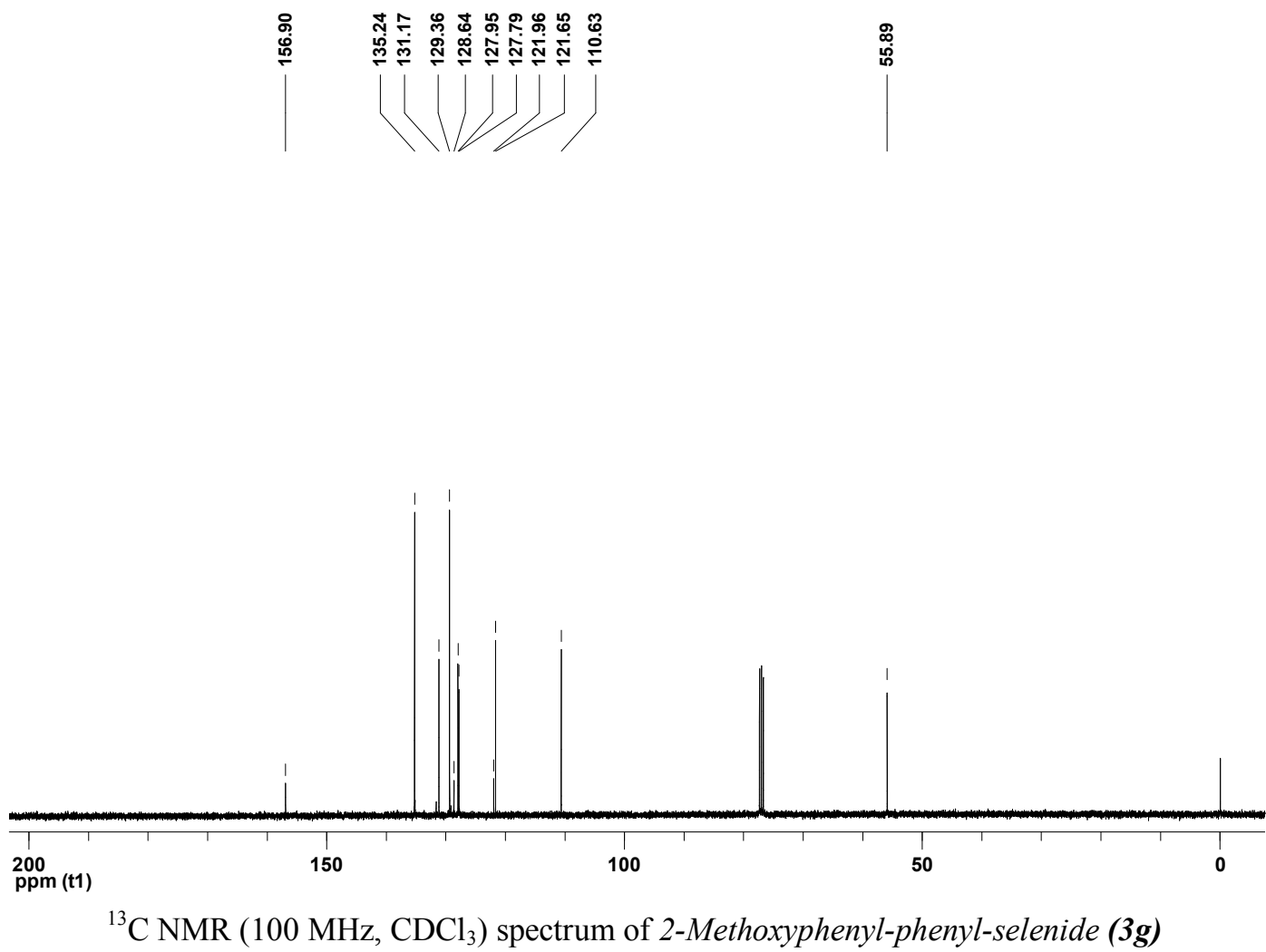

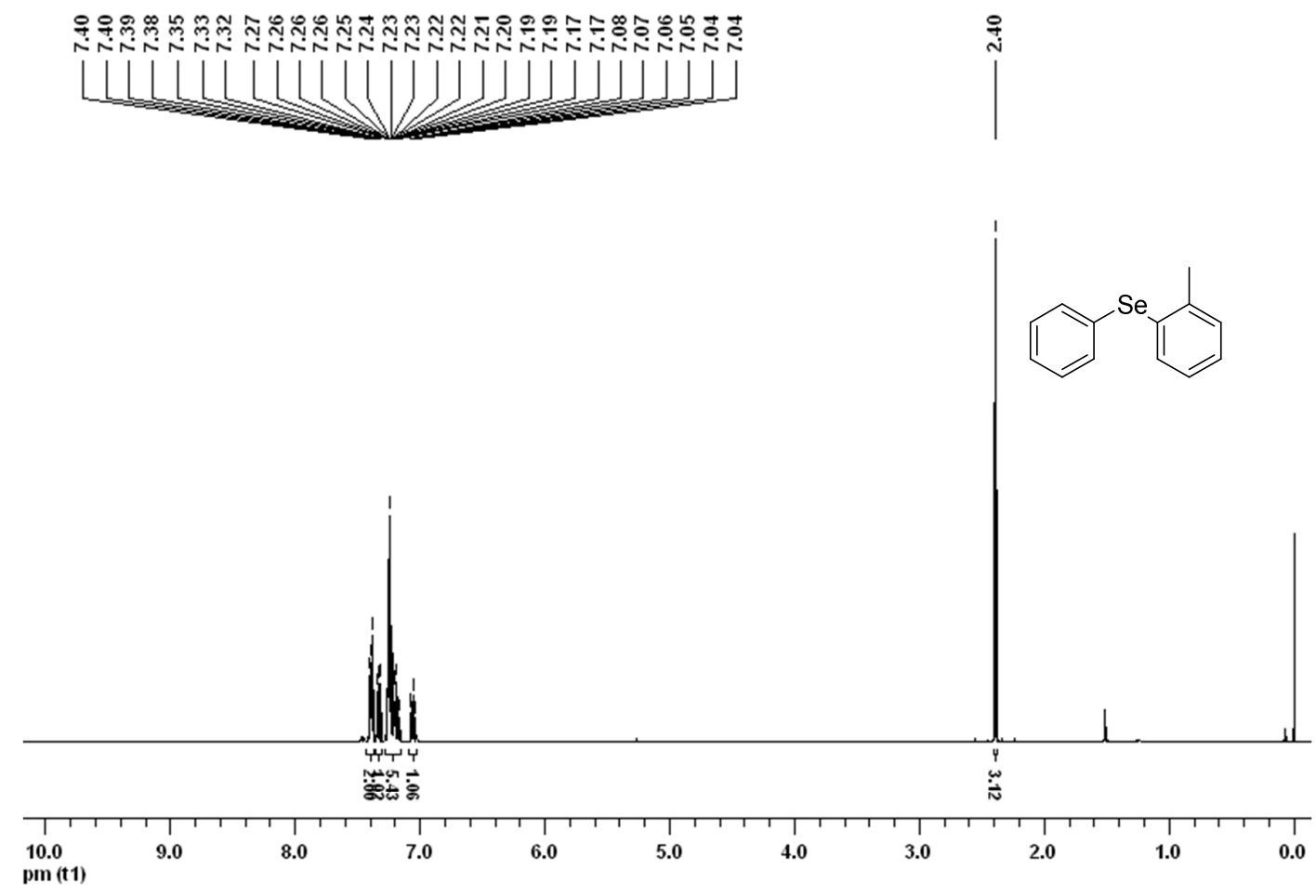

${ }^{1} \mathrm{H}$ NMR (400 MHz, $\mathrm{CDCl}_{3}$ ) spectrum of 2-Tolyl-phenyl-selenide (3h)
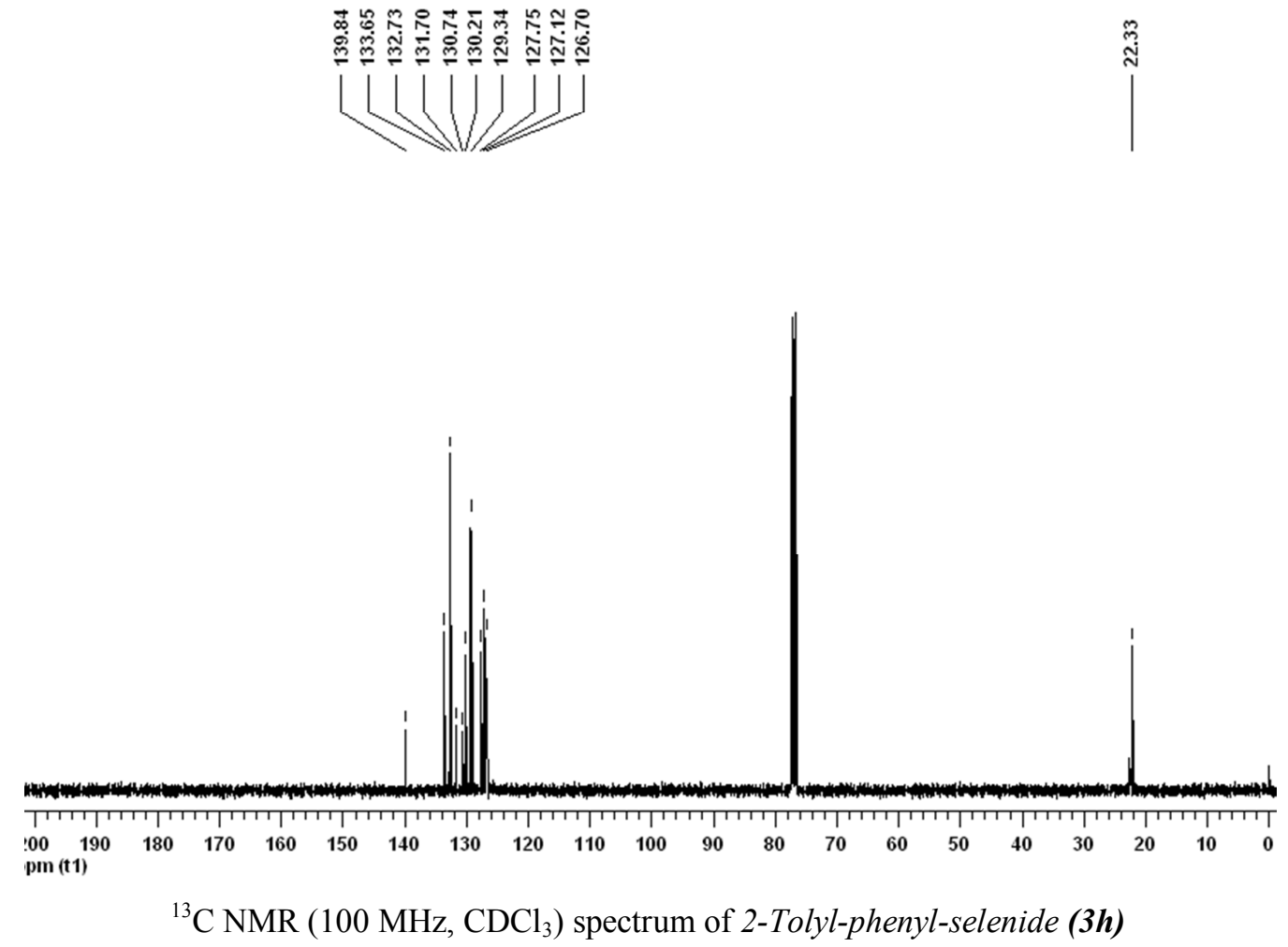

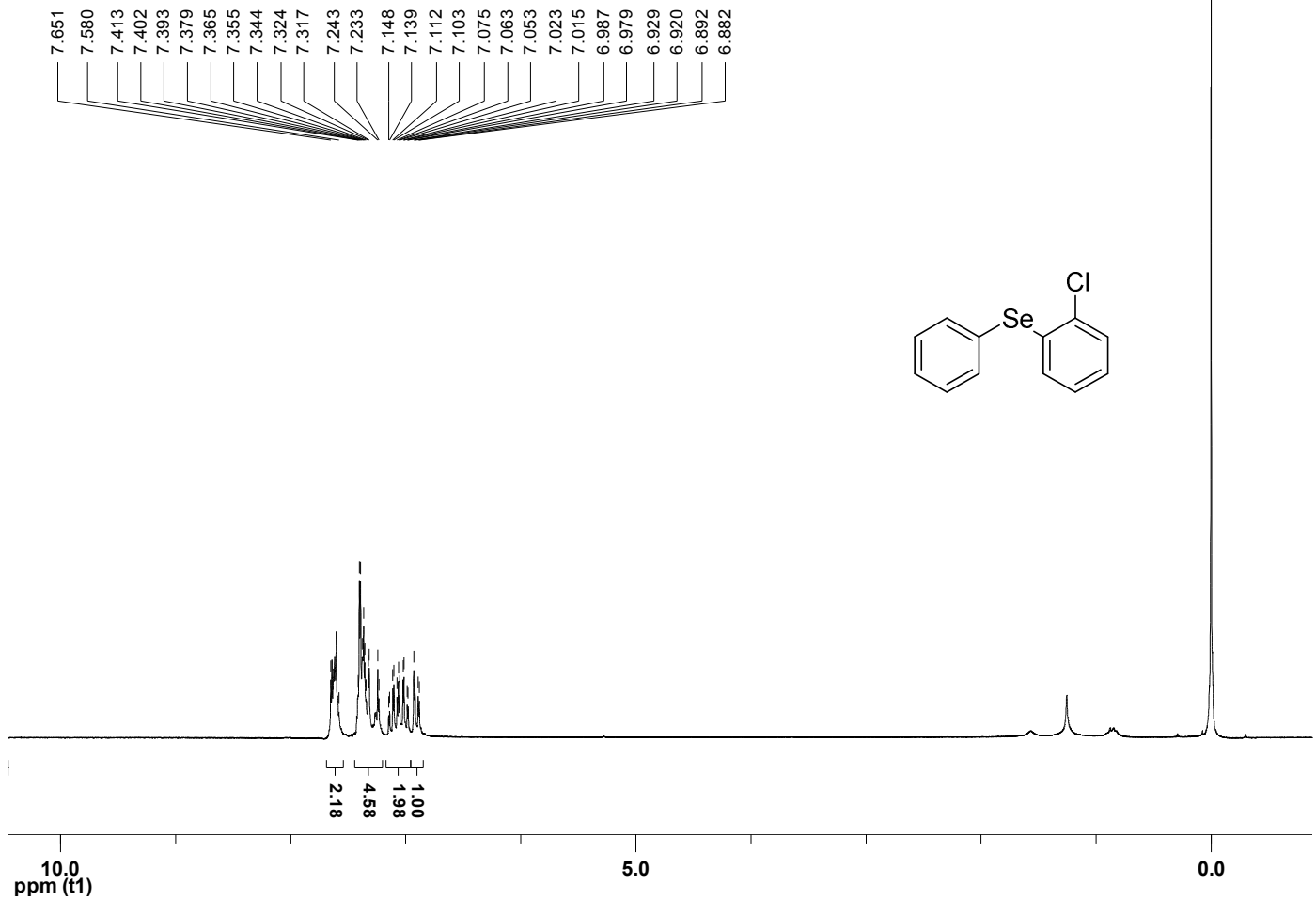

${ }^{1} \mathrm{H}$ NMR (400 MHz, $\mathrm{CDCl}_{3}$ ) spectrum of 2-Chlorophenyl-phenyl-selenide (3i)
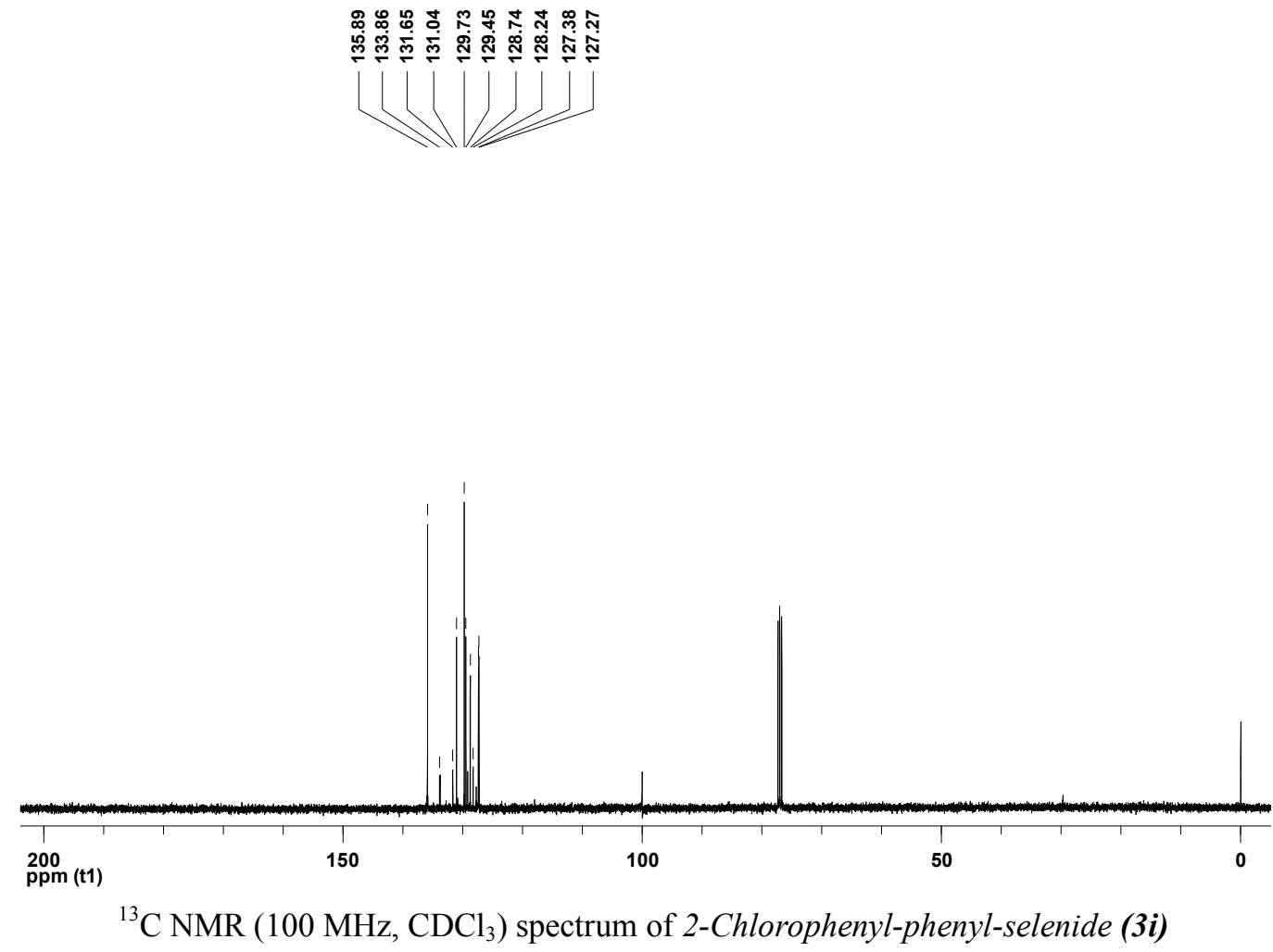


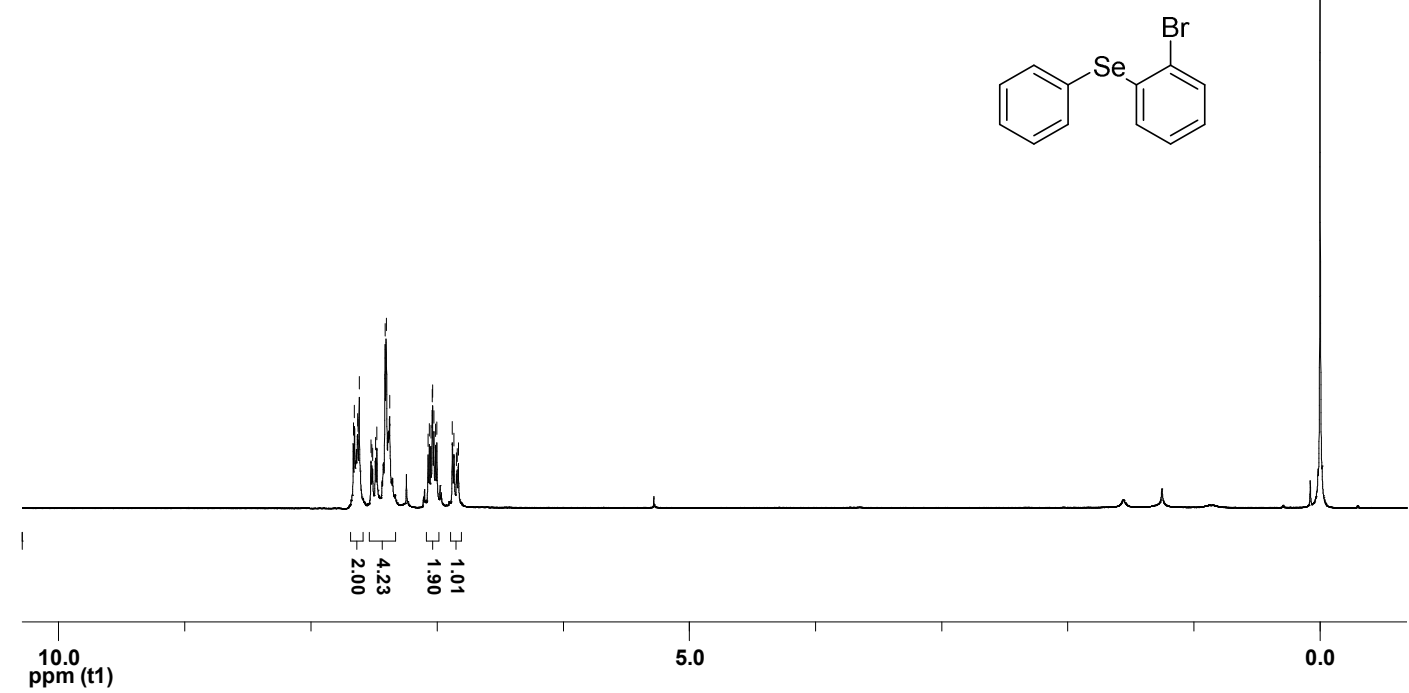

${ }^{1} \mathrm{H}$ NMR (400 MHz, $\mathrm{CDCl}_{3}$ ) spectrum of 2-Bromophenyl-phenyl-selenide (3j)

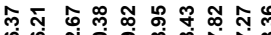

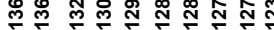
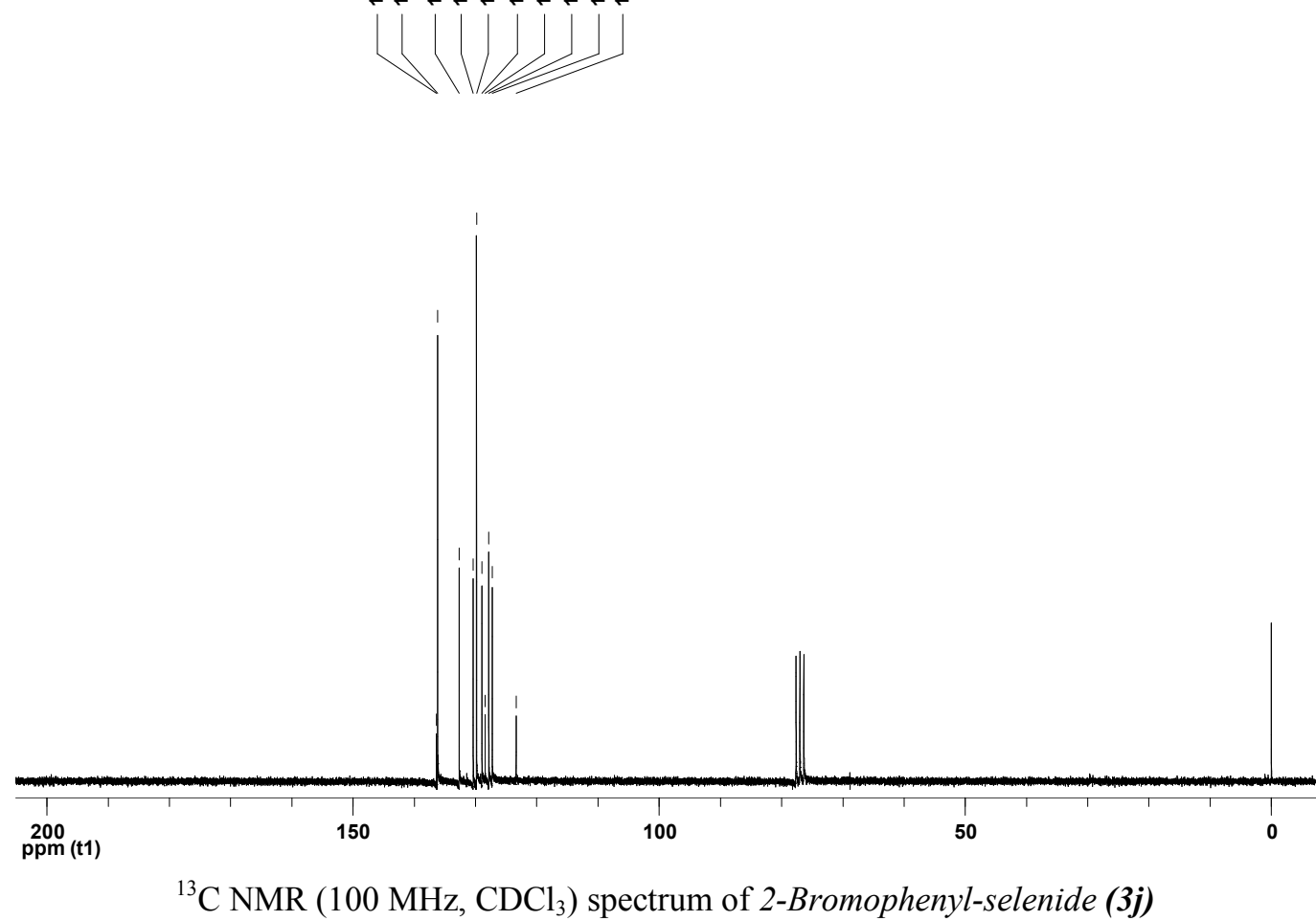


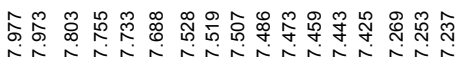

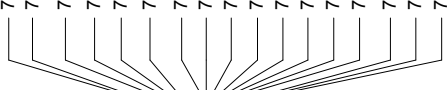

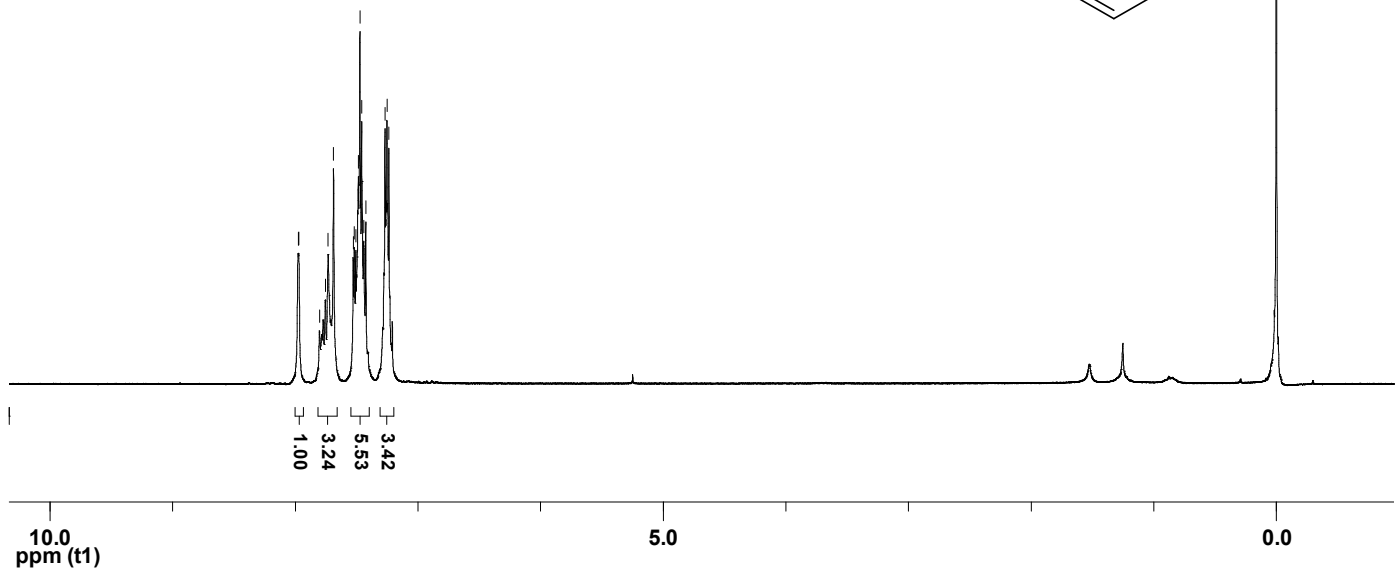

${ }^{1} \mathrm{H} \mathrm{NMR}\left(400 \mathrm{MHz}, \mathrm{CDCl}_{3}\right.$ ) spectrum of 2-Naphthyl-phenyl-selenide (3k)

ป็ ณ

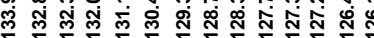
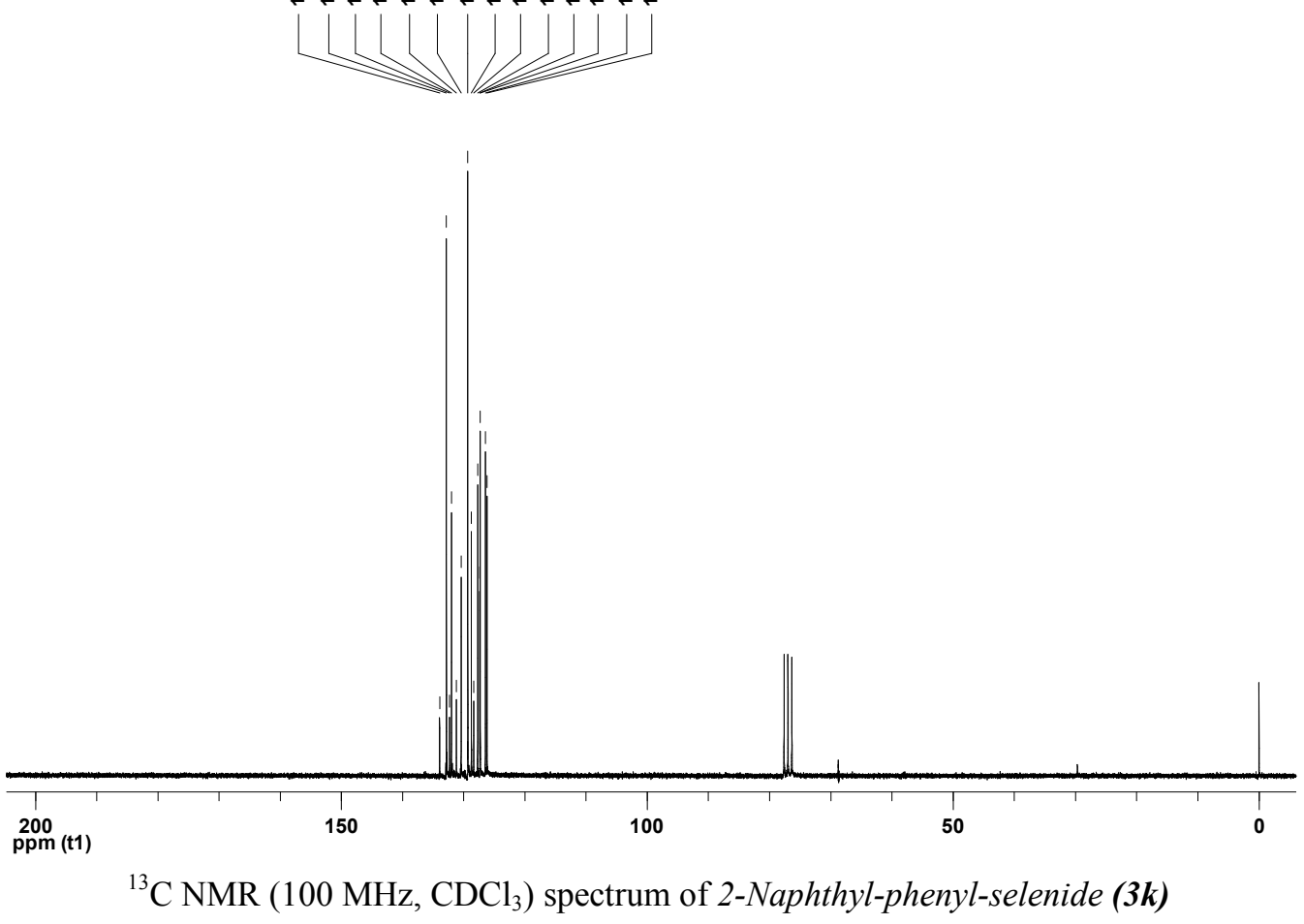
茴

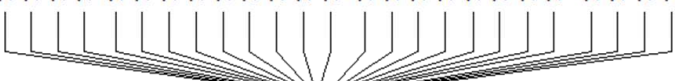

$\| y_{s}^{S e}$

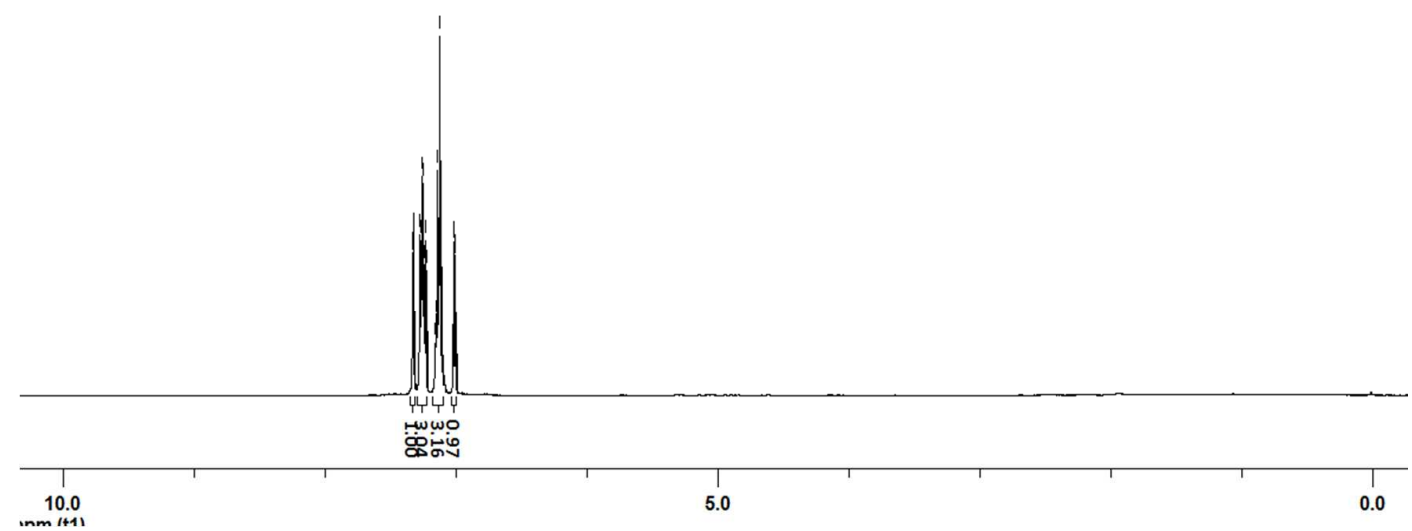

${ }^{1} \mathrm{H} \mathrm{NMR}\left(400 \mathrm{MHz}, \mathrm{CDCl}_{3}\right.$ ) spectrum of 3-thienyl-phenyl-selenide (3l)

$\infty$ กิ คำ

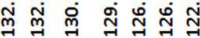
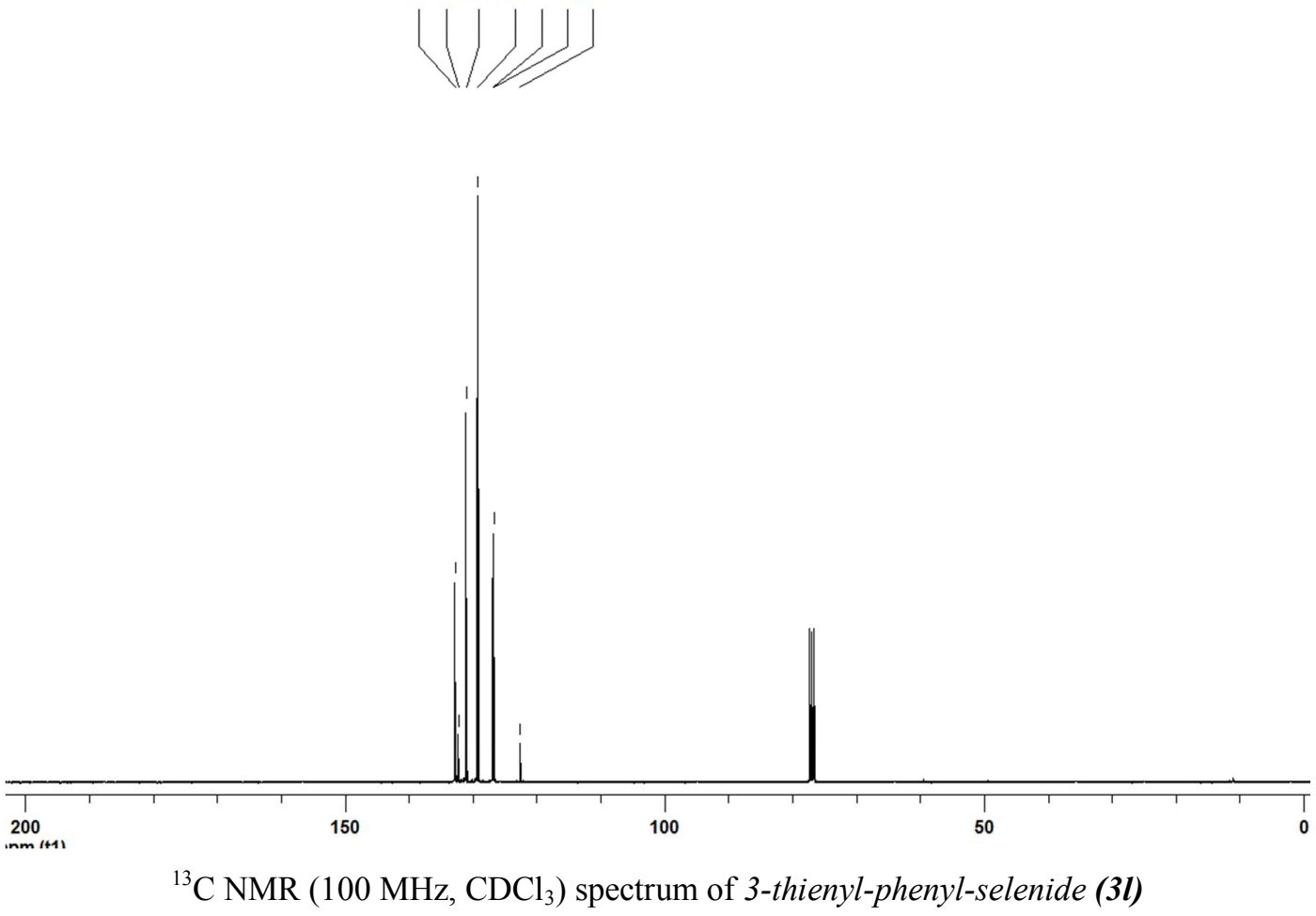


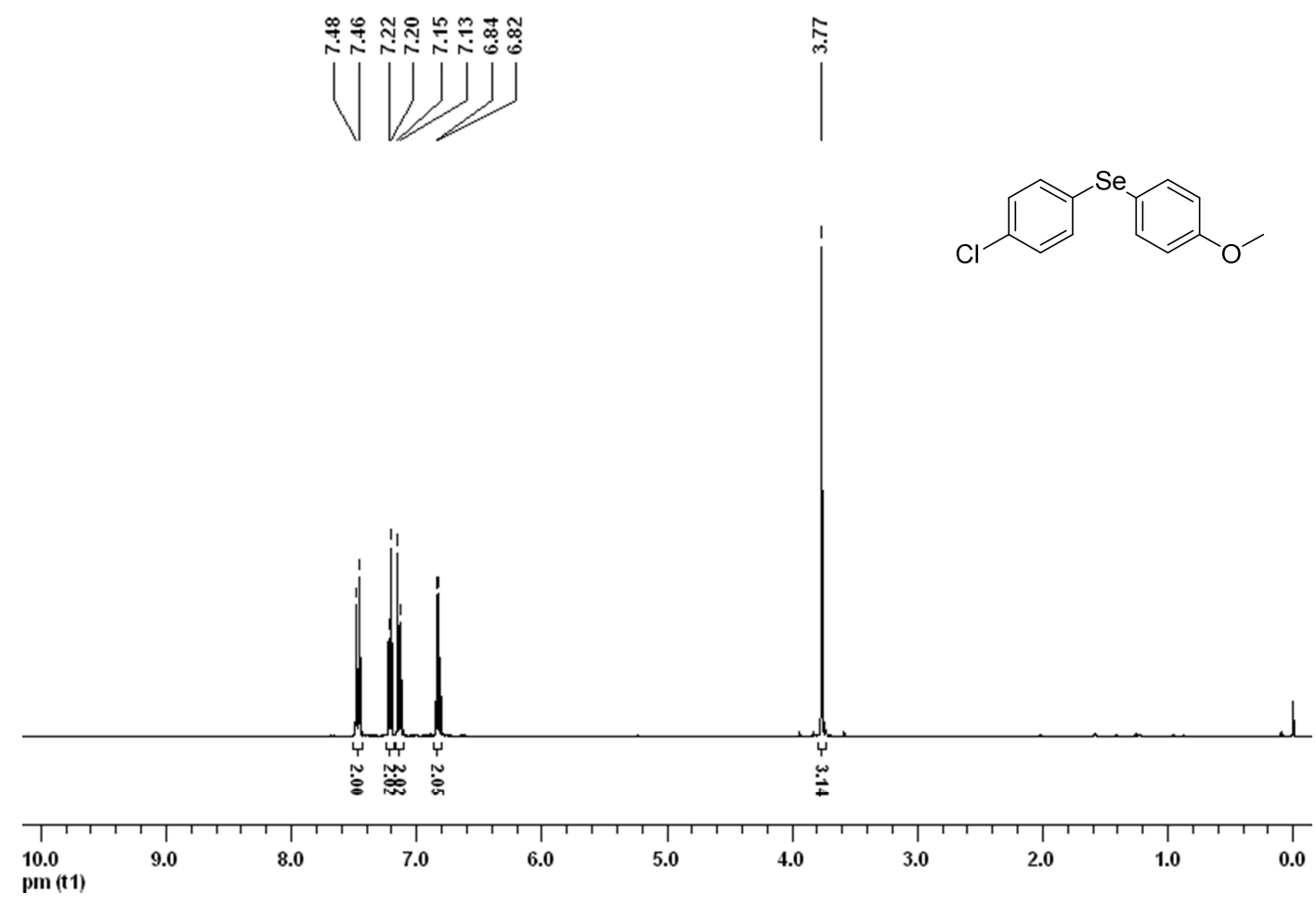

${ }^{1} \mathrm{H}$ NMR (400 MHz, $\mathrm{CDCl}_{3}$ ) spectrum of 4-Chlorophenyl-4-methoxylphenyl-selenide (3m)
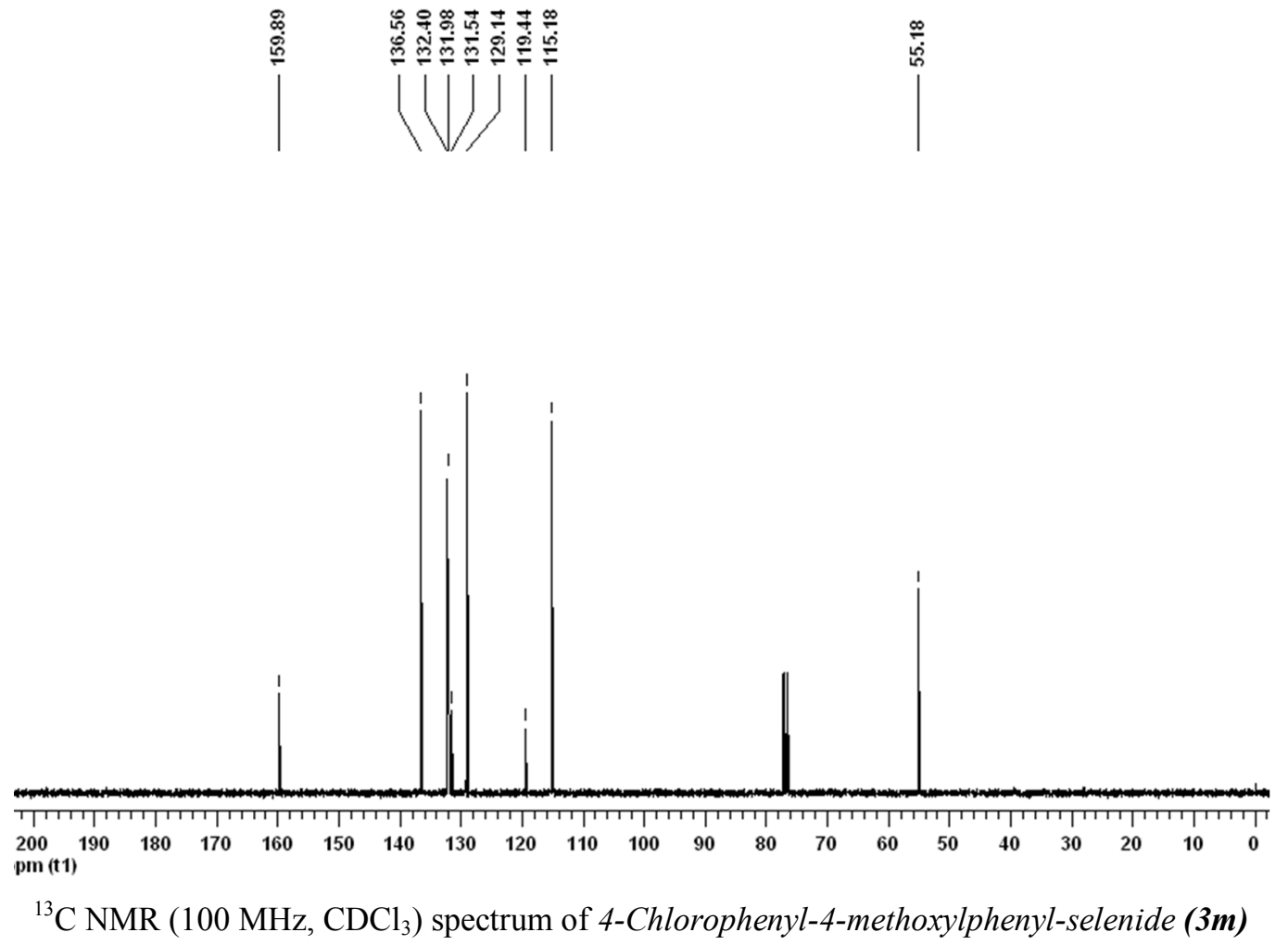


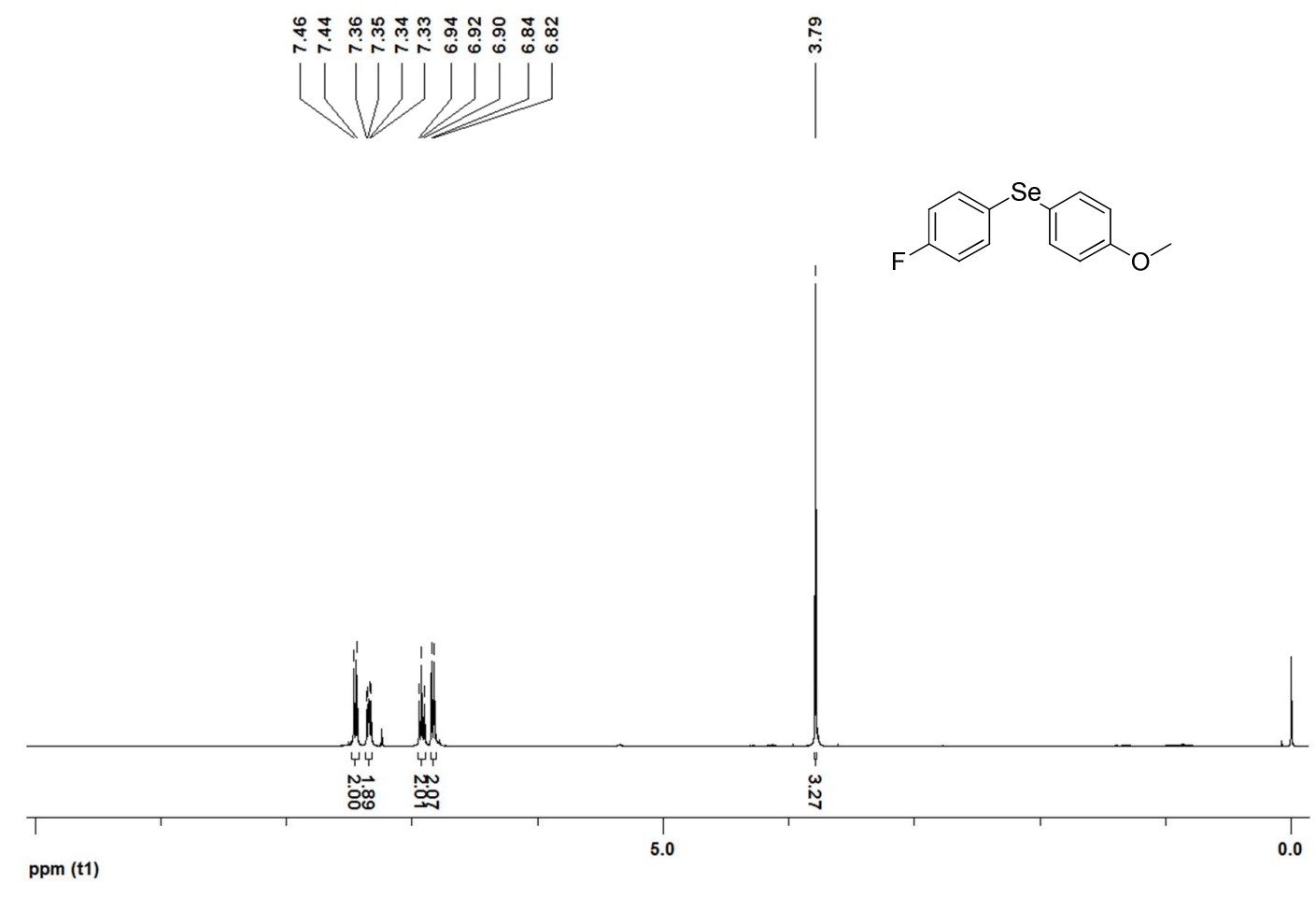

${ }^{1} \mathrm{H}$ NMR (400 MHz, $\mathrm{CDCl}_{3}$ ) spectrum of 4-Fluorophenyl-4-methoxylphenyl-selenide (3n)
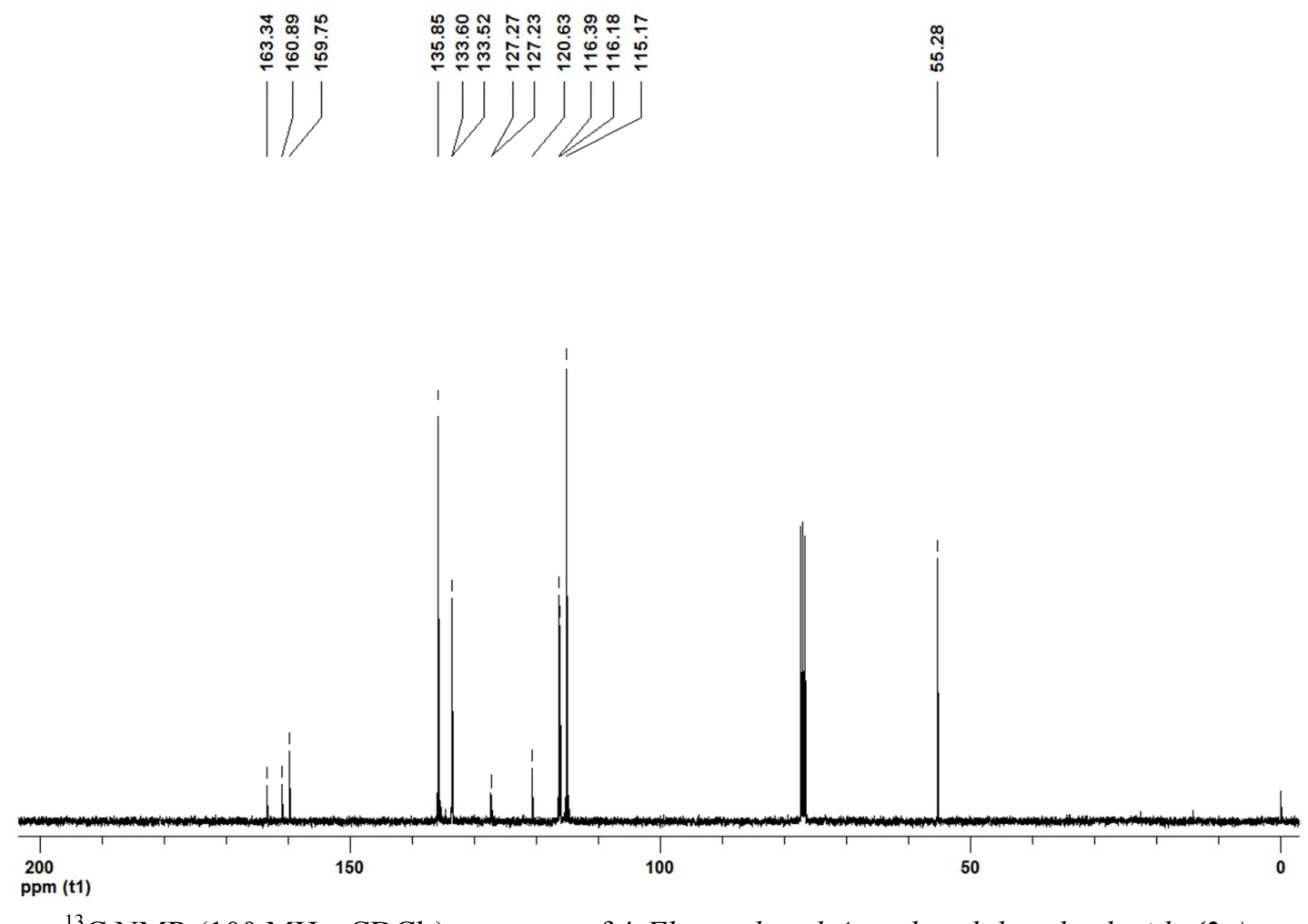

${ }^{13} \mathrm{C} \mathrm{NMR}\left(100 \mathrm{MHz}, \mathrm{CDCl}_{3}\right.$ ) spectrum of 4-Fluorophenyl-4-methoxylphenyl-selenide (3n) 


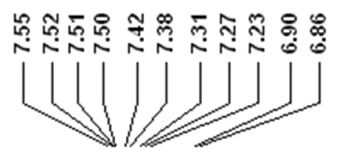

$$
\mid
$$

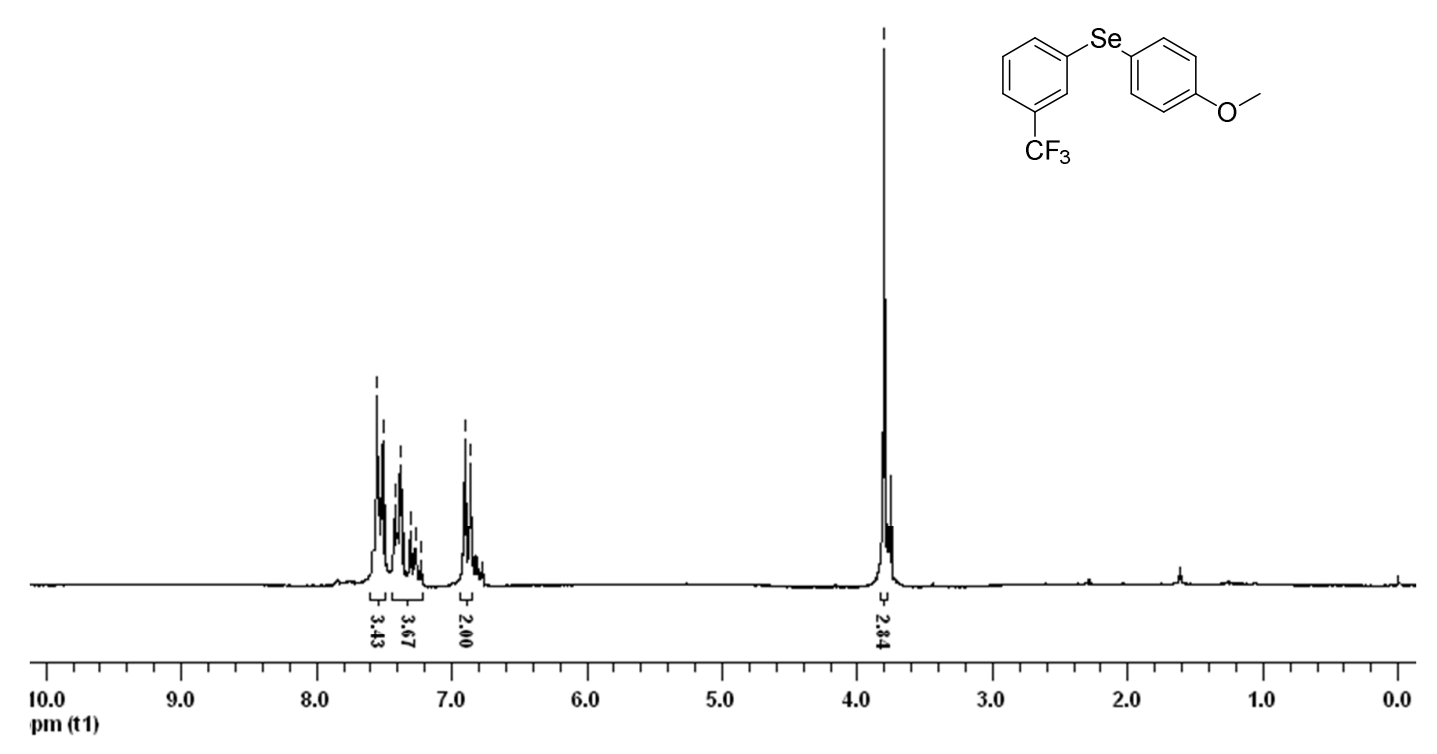

${ }^{1} \mathrm{H} \mathrm{NMR}\left(400 \mathrm{MHz}, \mathrm{CDCl}_{3}\right.$ ) spectrum of 3-(trifluoromethyl)phenyl-4-methoxylphenyl-selenide (3o)
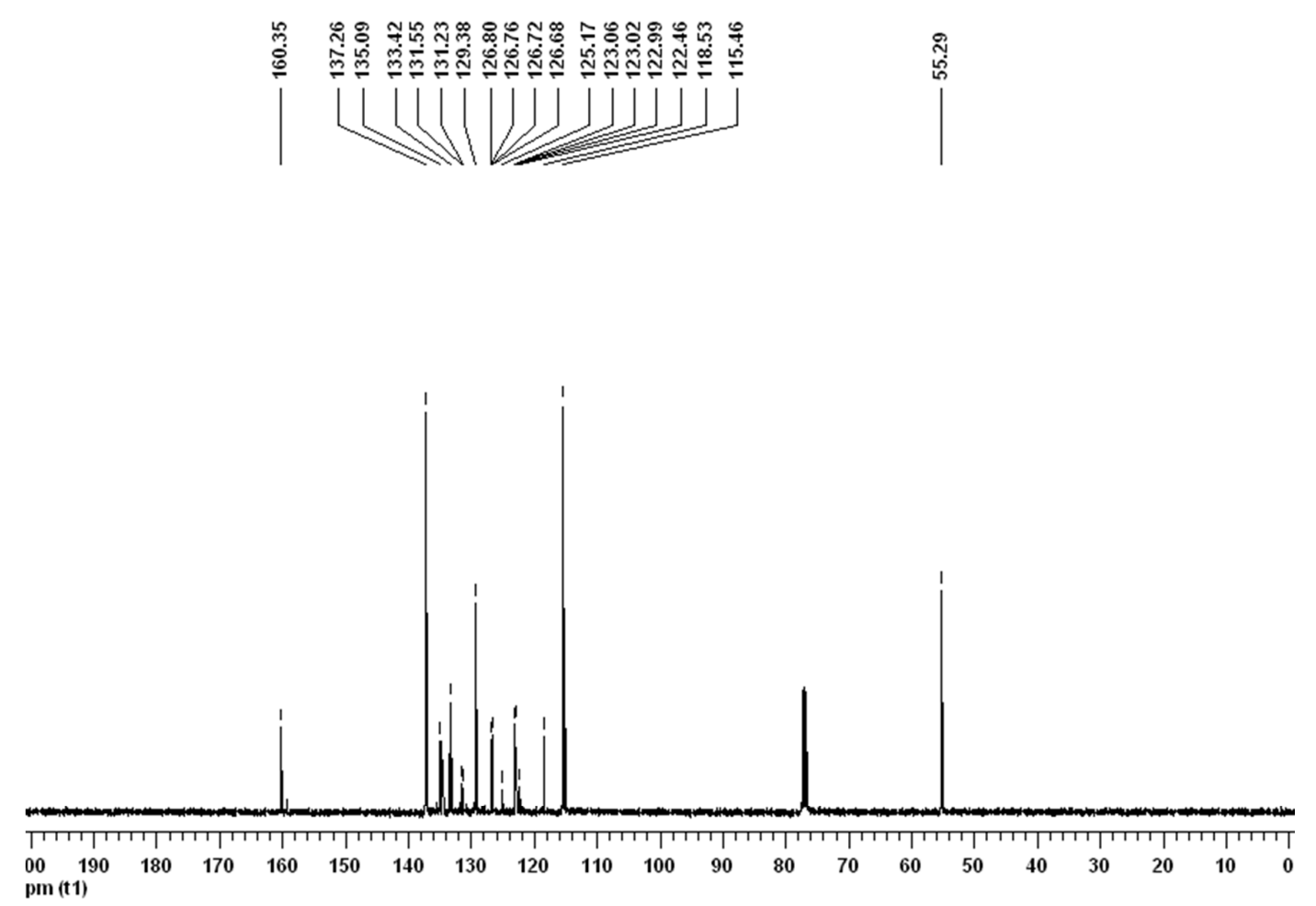

${ }^{13} \mathrm{C} \mathrm{NMR}\left(100 \mathrm{MHz}, \mathrm{CDCl}_{3}\right.$ ) spectrum of 3-(trifluoromethyl)phenyl-4-methoxylphenyl-selenide (3o) 


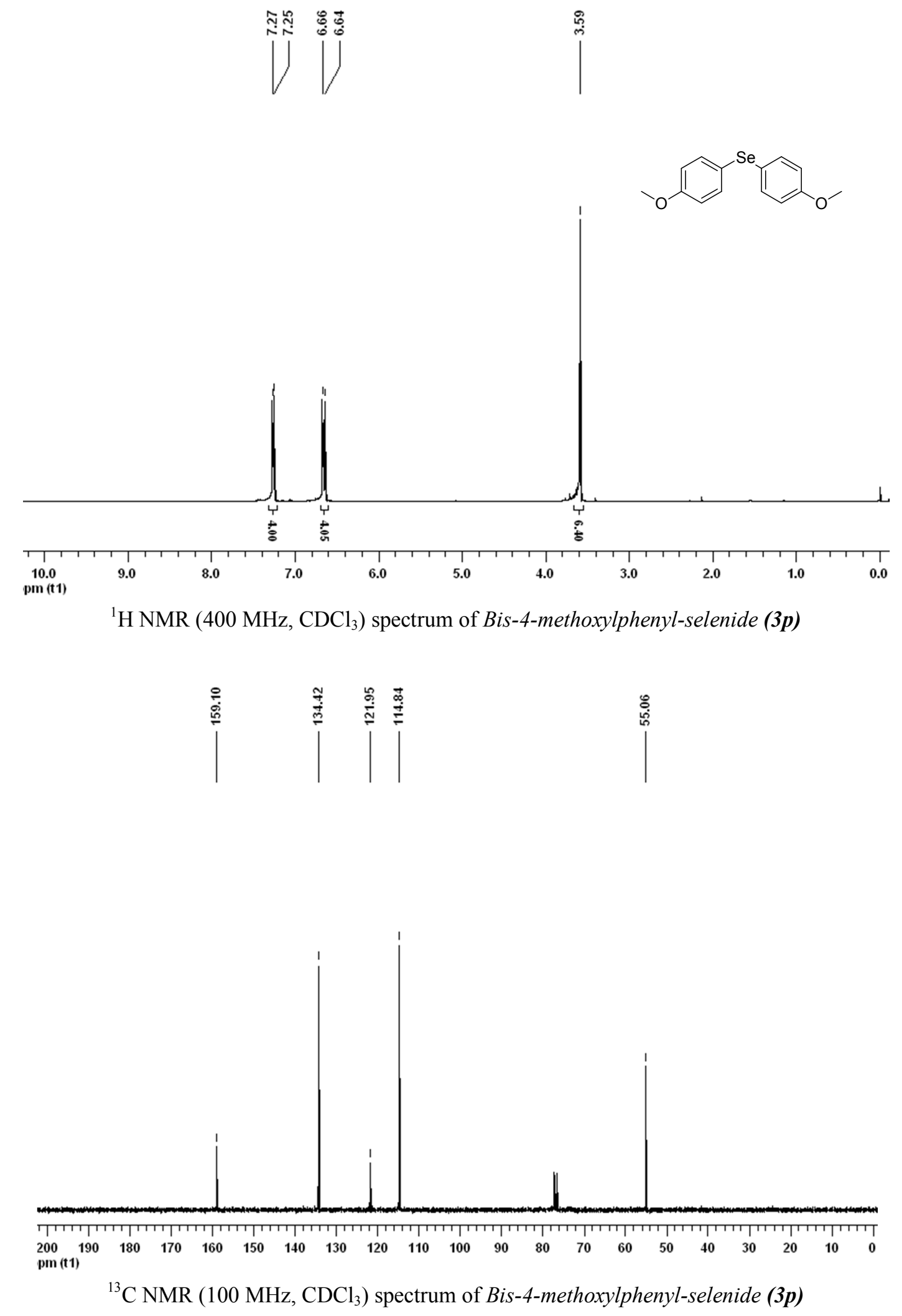




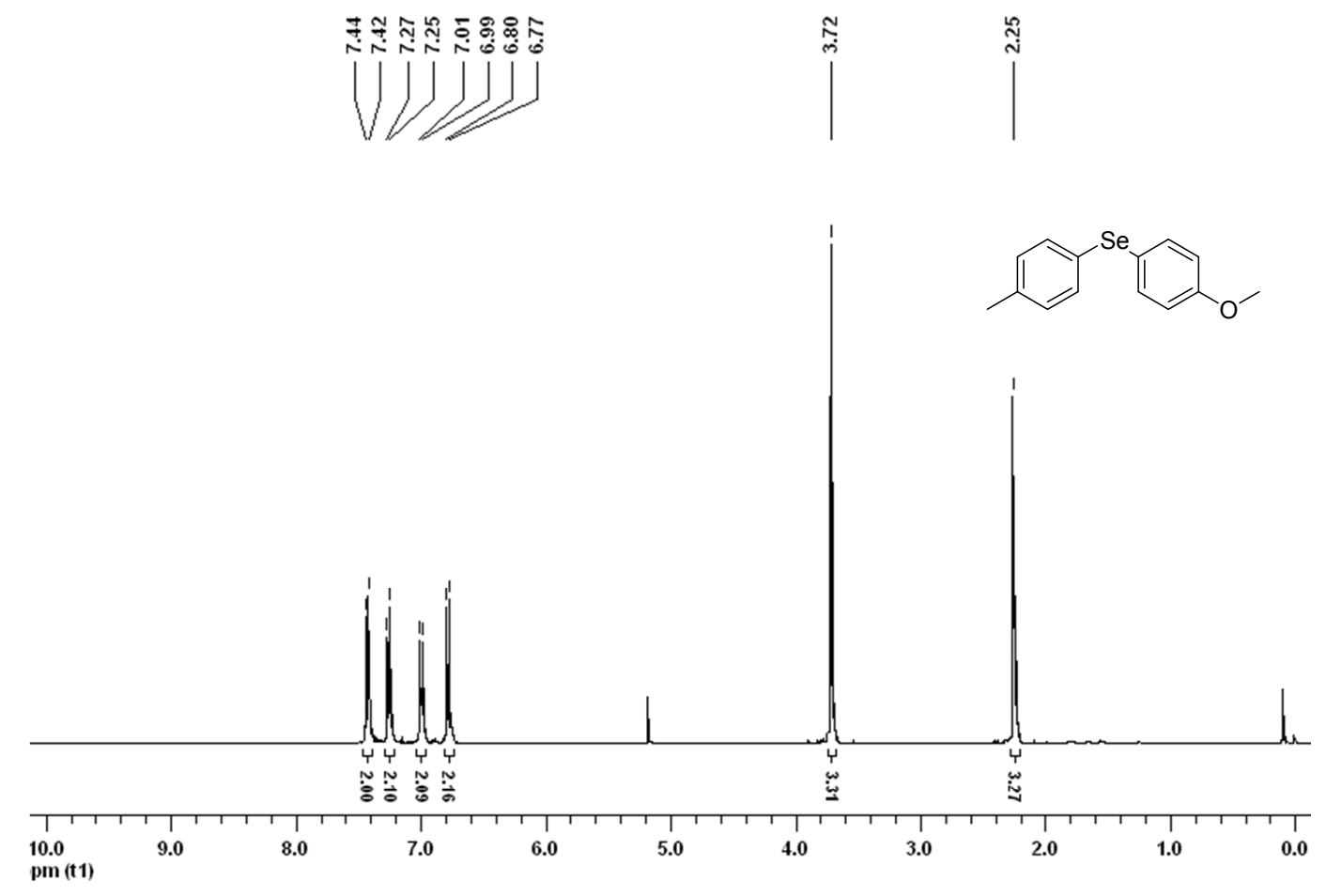

${ }^{1} \mathrm{H}$ NMR (400 MHz, $\mathrm{CDCl}_{3}$ ) spectrum of 4-Tolyl-4-methoxylphenyl-selenide (3q)

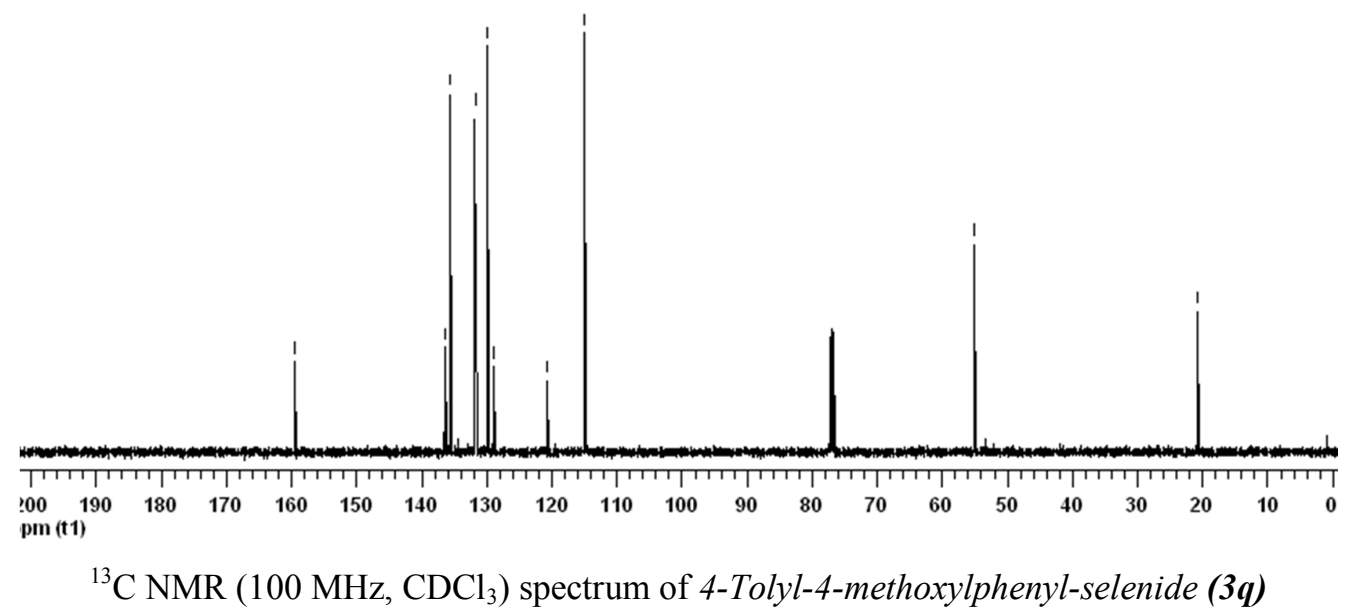



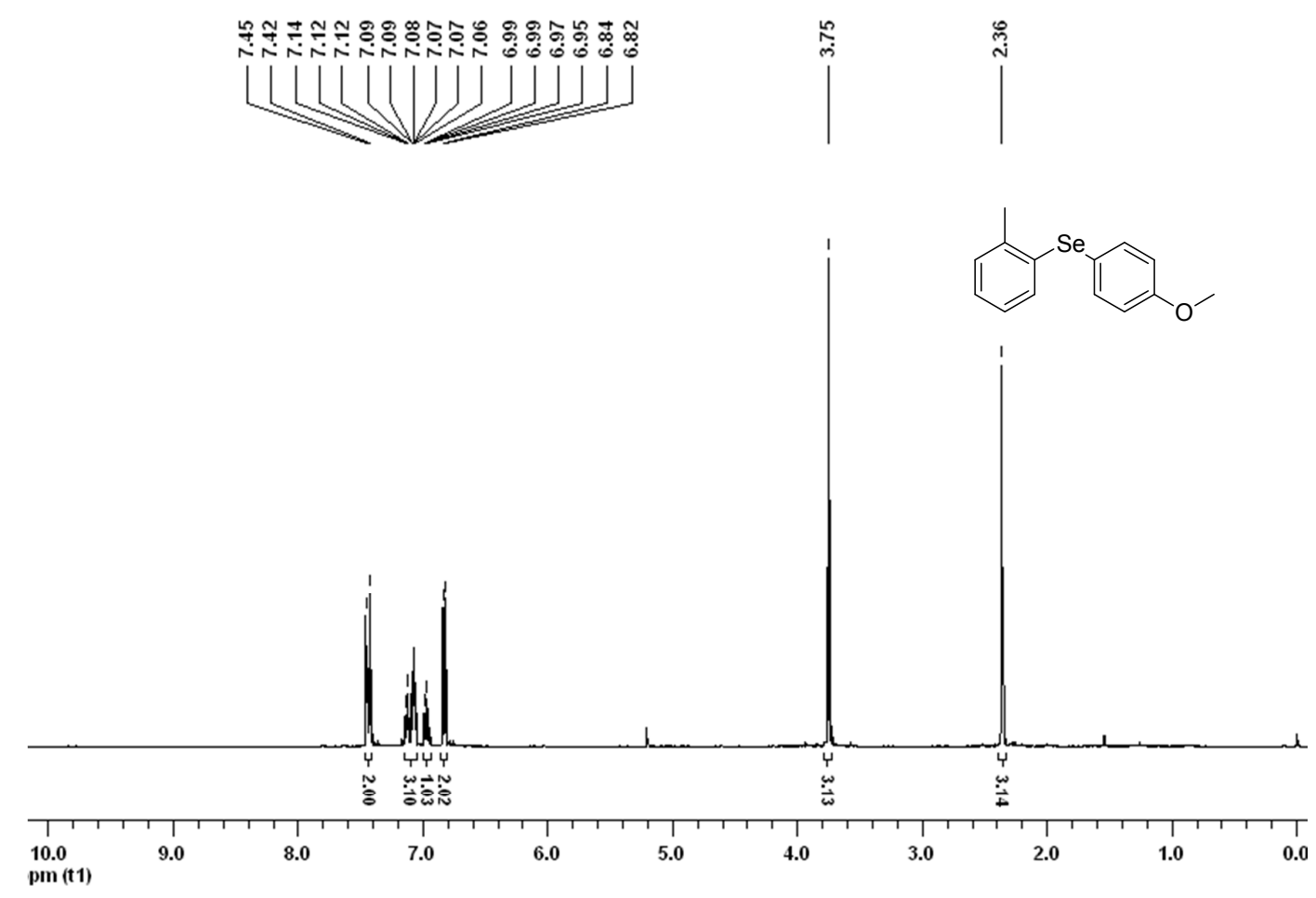

${ }^{1} \mathrm{H} \mathrm{NMR}\left(400 \mathrm{MHz}, \mathrm{CDCl}_{3}\right.$ ) spectrum of 2-Tolyl-4-methoxylphenyl-selenide (3r)
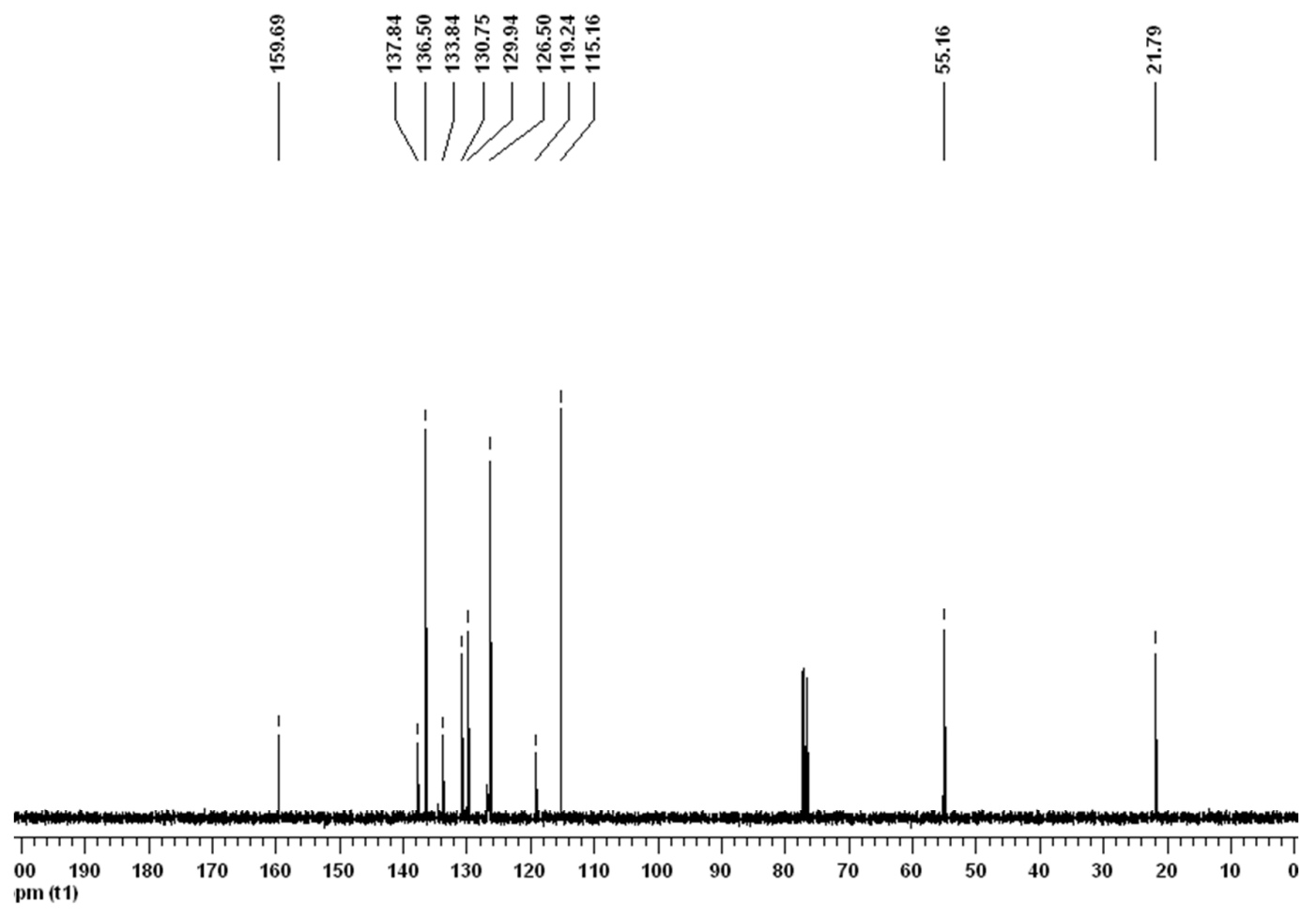

${ }^{13} \mathrm{C} \mathrm{NMR}\left(100 \mathrm{MHz}, \mathrm{CDCl}_{3}\right)$ spectrum of 2-Tolyl-4-methoxylphenyl-selenide (3r) 


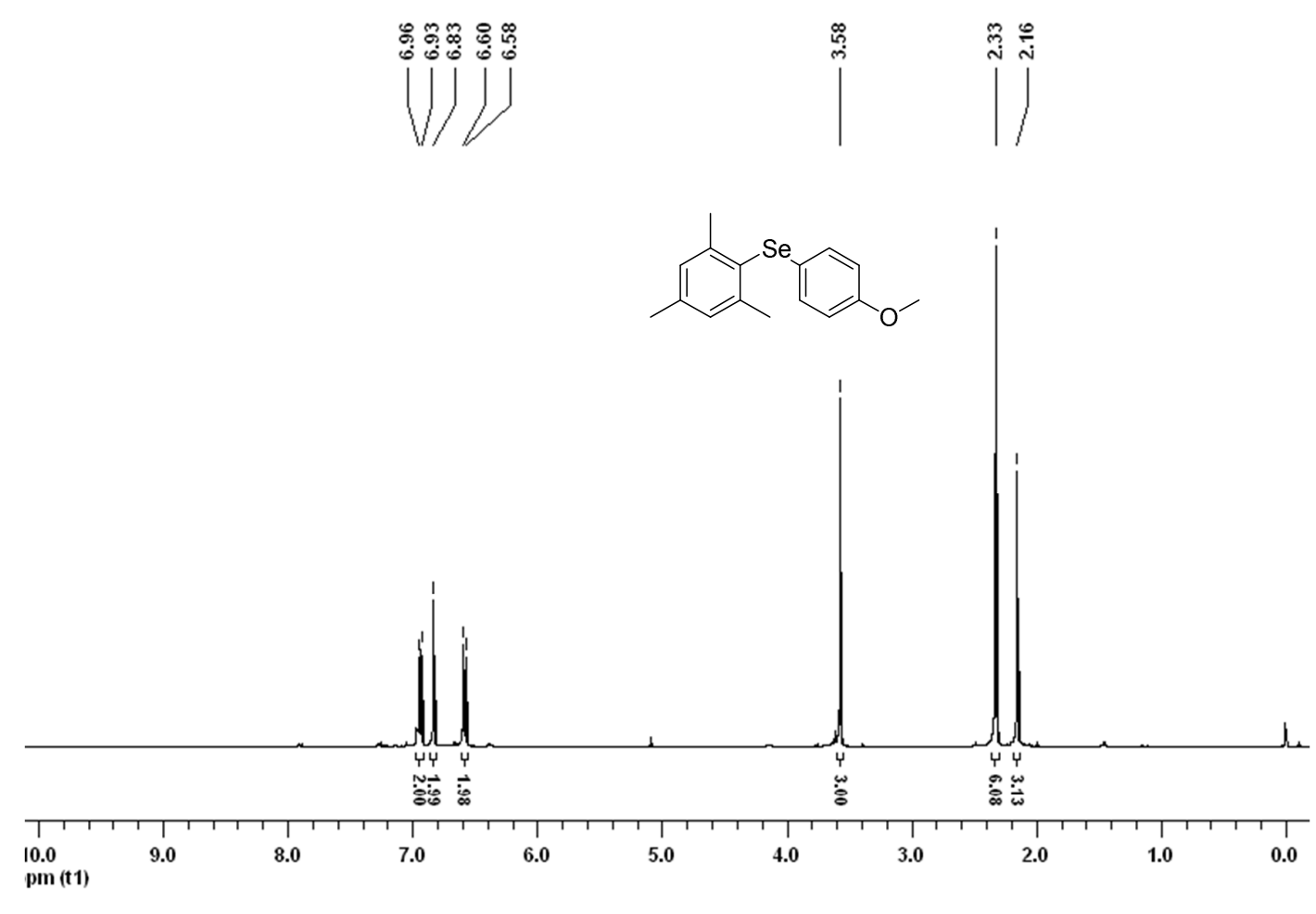

${ }^{1} \mathrm{H} \mathrm{NMR}\left(400 \mathrm{MHz}, \mathrm{CDCl}_{3}\right.$ ) spectrum of 2,4,6-(trimethyl)phenyl-4-methoxylphenyl-selenide (3s)
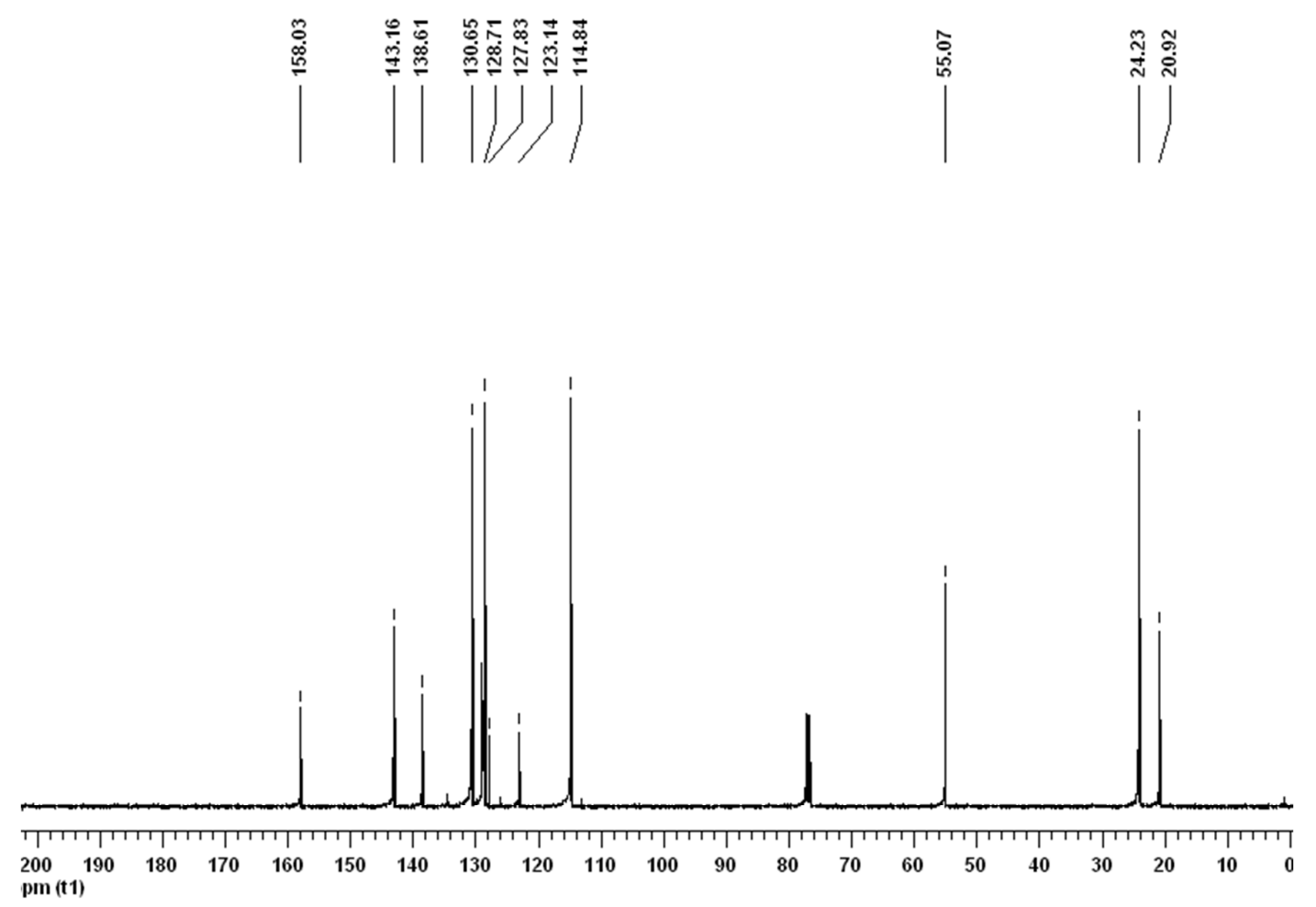

${ }^{13} \mathrm{C}$ NMR (100 MHz, $\mathrm{CDCl}_{3}$ ) spectrum of 2,4,6-(trimethyl)phenyl-4-methoxylphenyl-selenide (3s) S20 


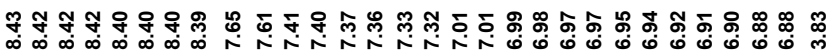
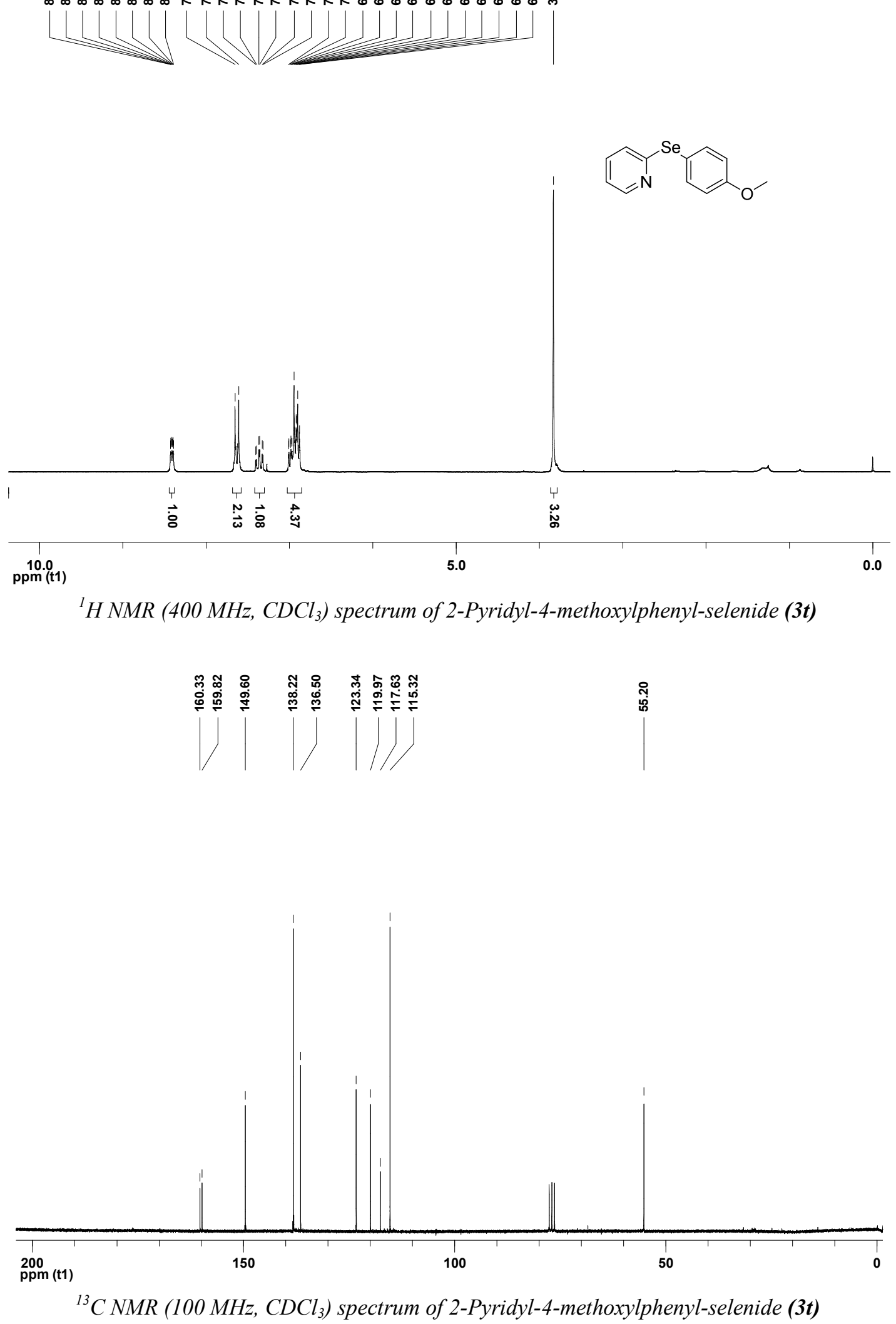

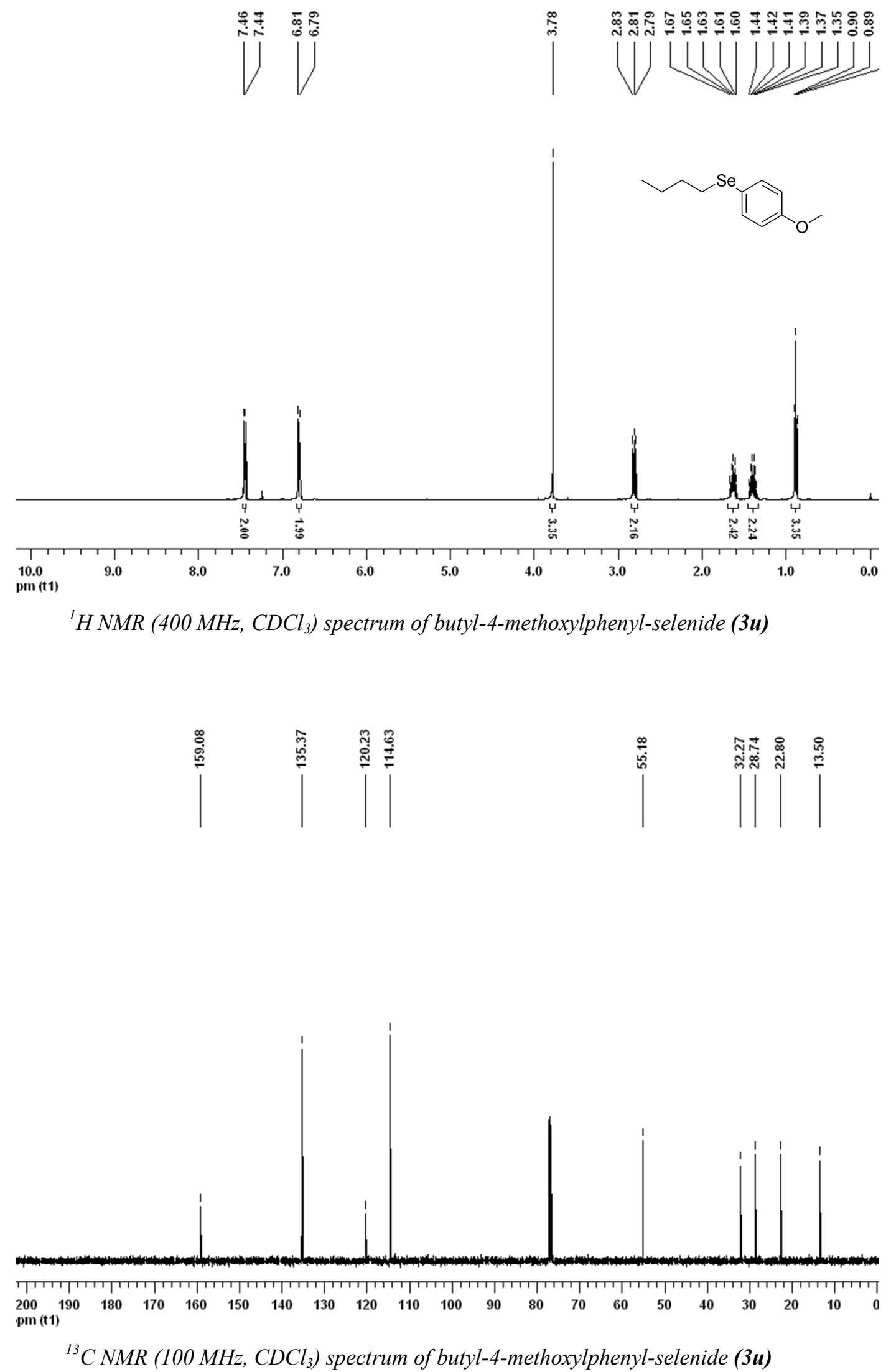

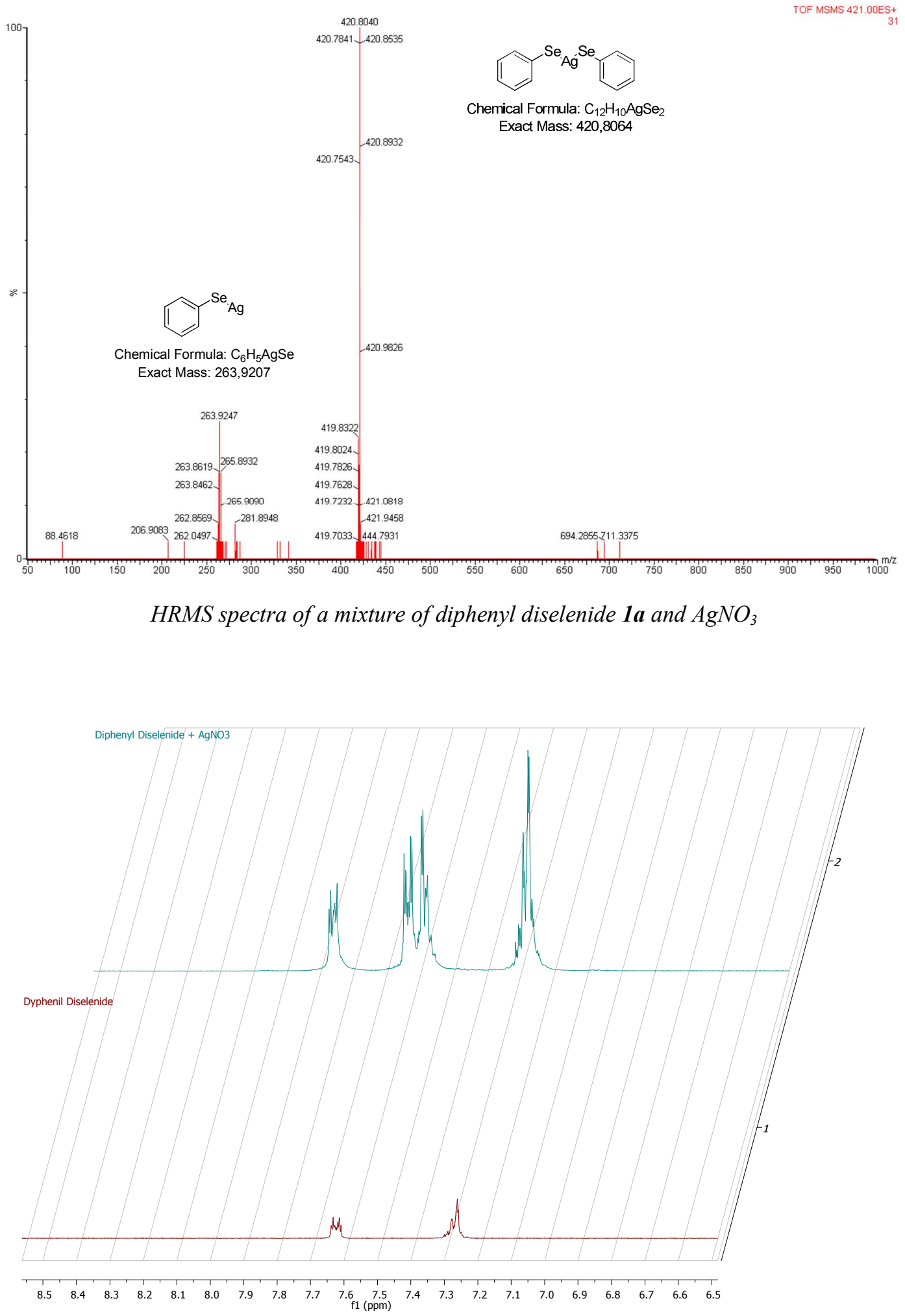

${ }^{1} \mathrm{H} \mathrm{NMR}(400 \mathrm{MHz})$ spectrum for diphenyl diselenide $1 \mathbf{a}$ and diphenyl diselenide $+\mathrm{AgNO}_{3}$ 


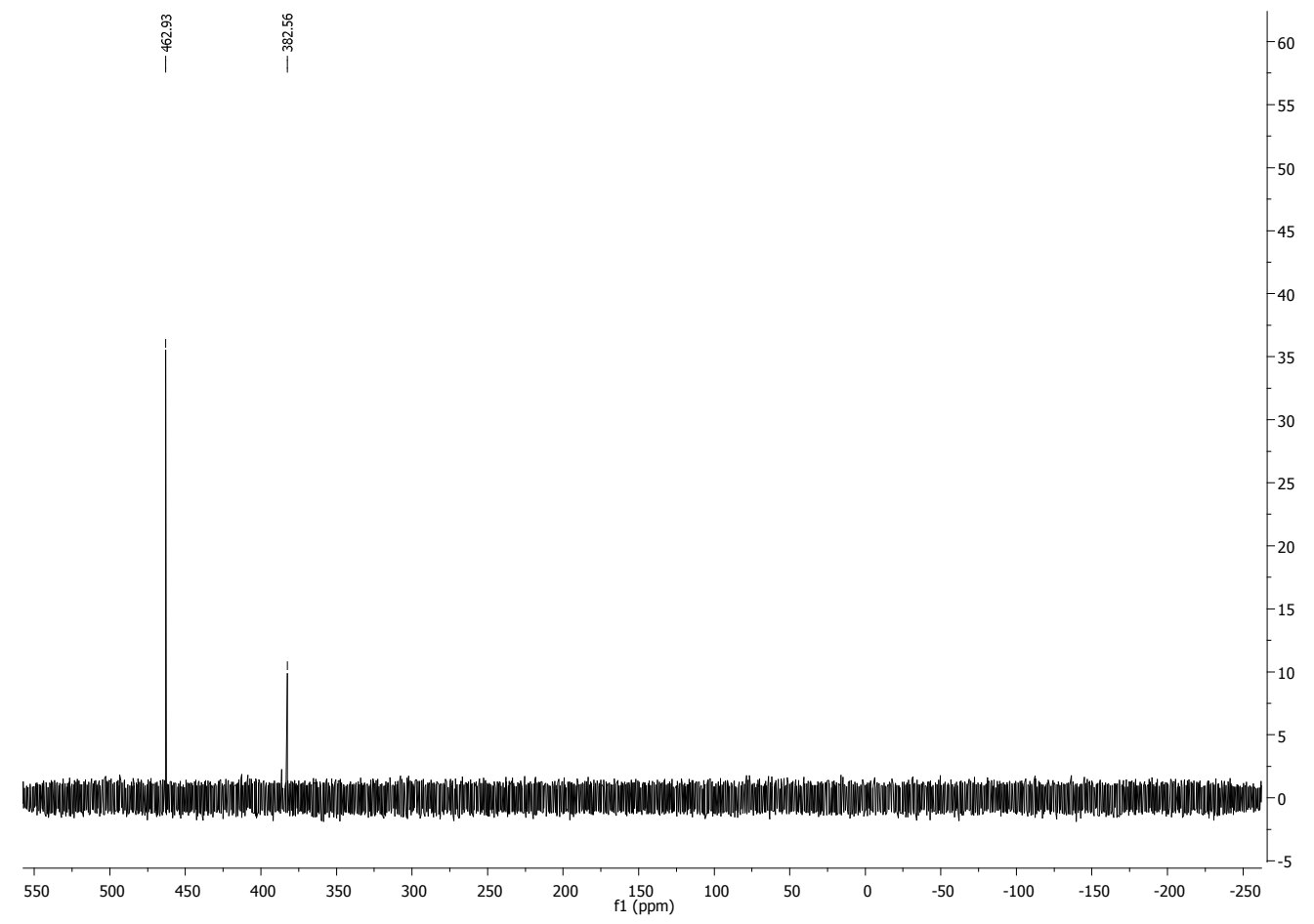

${ }^{77} \mathrm{Se} N \mathrm{NR}(57 \mathrm{MHz})$ of the mixture of diphenyl diselenide $1 \mathrm{a}$ and $\mathrm{AgNO}_{3}$ in $\mathrm{CDCl}_{3}$ 